\title{
Exoplanet Detection Techniques
}

\author{
Debra A. Fischer \\ Yale University \\ Andrew W. Howard \\ University of Hawai 'i at Manoa \\ Greg P. Laughlin \\ University of California at Santa Cruz \\ Bruce Macintosh \\ Stanford University \\ Suvrath Mahadevan \\ The Pennsylvania State University \\ Johannes Sahlmann \\ Université de Genève \\ Jennifer C. Yee \\ Harvard-Smithsonian Center for Astrophysics
}

\begin{abstract}
We are still in the early days of exoplanet discovery. Astronomers are beginning to model the atmospheres and interiors of exoplanets and have developed a deeper understanding of processes of planet formation and evolution. However, we have yet to map out the full complexity of multi-planet architectures or to detect Earth analogs around nearby stars. Reaching these ambitious goals will require further improvements in instrumentation and new analysis tools. In this chapter, we provide an overview of five observational techniques that are currently employed in the detection of exoplanets: optical and infrared (IR) Doppler measurements, transit photometry, direct imaging, microlensing, and astrometry. We provide a basic description of how each of these techniques works and discuss forefront developments that will result in new discoveries. We also highlight the observational limitations and synergies of each method and their connections to future space missions.
\end{abstract}

\section{INTRODUCTION}

Humans have long wondered whether other solar systems exist around the billions of stars in our galaxy. In the past two decades, we have progressed from a sample of one to a collection of hundreds of exoplanetary systems. Instead of an orderly solar nebula model, we now realize that chaos rules the formation of planetary systems. Gas giant planets can migrate close to their stars. Small rocky planets are abundant and dynamically pack the inner orbits. Planets circle outside the orbits of binary star systems. The diversity is astonishing.

Several methods for detecting exoplanets have been developed: Doppler measurements, transit observations, microlensing, astrometry, and direct imaging. Clever in- novations have advanced the precision for each of these techniques; however, each of the methods have inherent observational incompleteness. The lens through which we detect exoplanetary systems biases the parameter space that we can see. For example, Doppler and transit techniques preferentially detect planets that orbit closer to their host stars and are larger in mass or size, while microlensing, astrometry, and direct imaging are more sensitive to planets in wider orbits. In principle, the techniques are complementary; in practice, they are not generally applied to the same sample of stars, so our detection of exoplanet architectures has been piecemeal. The explored parameter space of exoplanet systems is a patchwork quilt that still has several missing squares. 


\section{THE DOPPLER TECHNIQUE}

\subsection{Historical Perspective}

The first Doppler-detected planets were met with skepticism. Campbell et al. (1988) identified variations in the residual velocities of $\gamma \mathrm{Ceph}$, a component of a binary star system, but attributed them to stellar activity signals until additional data confirmed it as a planet 15 years later (Hatzes et al., 2003). Latham et al. (1989) detected a Doppler signal around HD 114762 with an orbital period of $84 \mathrm{~d}$ and a mass $M_{P} \sin i=11 M_{\text {Jup }}$. Since the orbital inclination was unknown, they expected that the mass could be significantly larger and interpreted their data as a probable brown dwarf. When Mayor and Queloz (1995) modeled a Doppler signal in their data for the Sun-like star, 51 Pegasi, as a Jupitermass planet in a 4.23-d orbit, astronomers wondered if this could be a previously unknown mode of stellar oscillations (Gray, 1997) or nonradial pulsations (Hatzes et al., 1997). The unexpected detection of significant eccentricity in exoplanet candidates further raised doubts among astronomers, who argued that although stars existed in eccentric orbits, planets should reside in circular orbits (Black, 1997). It was not until the first transiting planet (Henry et al., 2000; Charbonneau et al., 2000) and the first multi-planet system (Butler et al., 1999) were detected (almost back-to-back) that the planet interpretation of the Doppler velocity data was almost unanimously accepted.

The Doppler precision improved from about $10 \mathrm{~m} \mathrm{~s}^{-1}$ in 1995 to $3 \mathrm{~m} \mathrm{~s}^{-1}$ in 1998 , and then to about $1 \mathrm{~m} \mathrm{~s}^{-1}$ in 2005 when the High Accuracy Radial velocity Planet Searcher (HARPS) was commissioned (Mayor et al., 2003). A Doppler precision of $1 \mathrm{~m} \mathrm{~s}^{-1}$ corresponds to shifts of stellar lines across 1/1000th of a CCD pixel. This is a challenging measurement that requires high signal-to-noise $(\mathrm{S} / \mathrm{N})$, high resolution, and large spectral coverage. Echelle spectrometers typically provide these attributes and have served as the workhorse instruments for Doppler planet searches.

Figure 1 shows the detection history for planets identified with Doppler surveys (planets that also are observed to transit their host star are shown in light gray). The first planets were similar in mass to Jupiter and there has been a striking decline in the lower envelope of detected planet mass with time as instrumentation improved.

\subsection{Radial Velocity Measurements}

The Doppler technique measures the reflex velocity that an orbiting planet induces on a star. Because the star-planet interaction is mediated by gravity, more massive planets result in larger and more easily detected stellar velocity amplitudes. It is also easier to detect close-in planets, both because the gravitational force increases with the square of the distance and because the orbital periods are shorter and therefore more quickly detected. Lovis and Fischer (2011) provide a detailed discussion of the technical aspects of Doppler analysis with both an iodine cell and a thorium- argon simultaneous reference source.

The radial velocity semi-amplitude, $\mathrm{K}_{1}$, of the star can be expressed in units of $\mathrm{cm} \mathrm{s}^{-1}$ with the planet mass in units of $\mathrm{M}_{\oplus}$

$$
\mathrm{K}_{*}=\frac{8.95 \mathrm{~cm} \mathrm{~s}^{-1}}{\sqrt{1-\mathrm{e}^{2}}} \frac{\mathrm{M}_{\mathrm{P}} \sin \mathrm{i}}{\mathrm{M}_{\oplus}}\left(\frac{\mathrm{M}_{*}+\mathrm{M}_{\mathrm{P}}}{\mathrm{M}_{\odot}}\right)^{-2 / 3}\left(\frac{\mathrm{P}}{\mathrm{yr}}\right)^{-1 / 3}
$$

The observed parameters (velocity semi-amplitude $\mathrm{K}_{*}$, orbital period P, eccentricity e, and orientation angle $\omega$ ) are used to calculate a minimum mass of the planet $M_{P} \sin i$ if the mass of the star $\mathrm{M}_{*}$ is known. The true mass of the planet is unknown because it is modulated by the unknown inclination. For example, if the orbital inclination is $30^{\circ}$, the true mass is a factor of $2 \times$ the Doppler-derived $M_{P} \sin i$. The statistical probability that the orbit inclination is within an arbitrary range $\mathrm{i}_{1}<\mathrm{I}<\mathrm{i}_{2}$ is given by

$$
\mathcal{P}_{\text {incl }}=\left|\cos \left(\mathrm{i}_{2}\right)-\cos \left(\mathrm{i}_{1}\right)\right|
$$

Thus, there is a roughly $87 \%$ probability that random orbital inclinations are between $30^{\circ}$ and $90^{\circ}$, or equivalently, an $87 \%$ probability that the true mass is within a factor of 2 of the minimum mass $\mathrm{M}_{\mathrm{P}} \sin \mathrm{i}$.

Radial velocity observations must cover one complete orbit in order to robustly measure the orbital period. As a result, the first detected exoplanets resided in short-period orbits. Doppler surveys that have continued for a decade or more (Fischer et al., 2014; Marmier et al., 2013) have been able to detect gas giant planets in Jupiter-like orbits.

\subsection{The Floor of the Doppler Precision}

An important question is whether the Doppler technique can be further improved to detect smaller planets at wider orbital radii. The number of exoplanets detected each year rose steadily until 2011 and has dropped precipitously after that year. This is due in part to the fact that significant telescope time has been dedicated to transit follow-up and also because observers are working to extract the smallest possible planets, requiring more Doppler measurement points given current precision. Further gains in Doppler precision and productivity will require new instruments with greater stability as well as analytical techniques for decorrelating stellar noise.

Figure 2, reproduced from Pepe et al. (2011), shows an example of one of the lowest-amplitude exoplanets, detected with HARPS. The velocity semi-amplitude for this planet is $\mathrm{K}=0.769 \mathrm{~m} \mathrm{~s}^{-1}$ and the orbital period is $58.43 \mathrm{~d}$. The data comprised 185 observations spanning $7.5 \mathrm{yr}$. The residual velocity scatter after fitting for the planet was reported to be $0.77 \mathrm{~m} \mathrm{~s}^{-1}$, showing that high precision can be achieved with many data points to beat down the single measurement precision.

One promising result suggests that it may be possible for stable spectrometers to average over stellar noise signals 


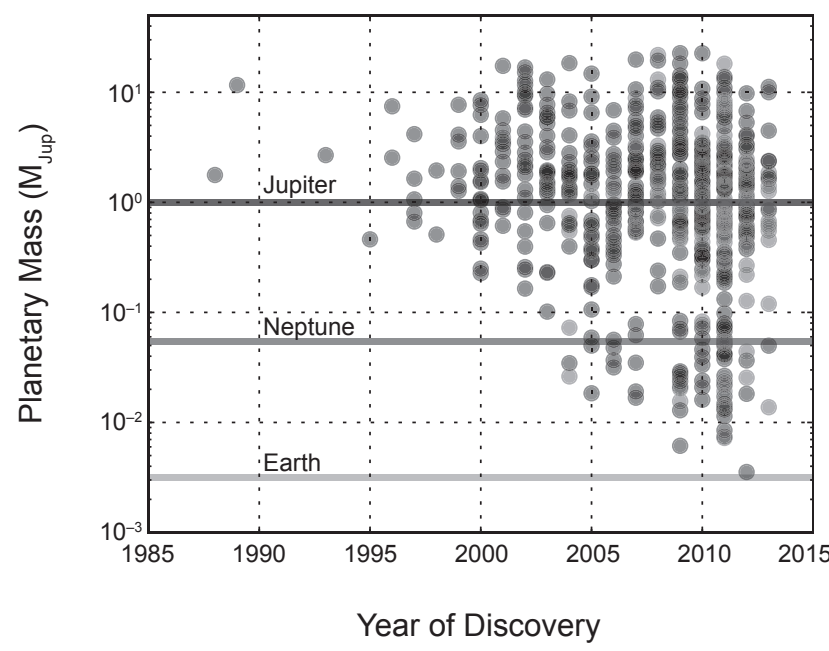

Fig. 1. Planet mass is plotted as a function of the year of discovery. The color coding is black for planets with no known transit, whereas light gray is planets that do transit.

and reach precisions below $0.5 \mathrm{~m} \mathrm{~s}^{-1}$, at least for some stars. After fitting for three planets in HD 20794, Pepe et al. (2011) found that the RMS of the residual velocities decreased from $0.8 \mathrm{~m} \mathrm{~s}^{-1}$ to $0.2 \mathrm{~m} \mathrm{~s}^{-1}$ as they binned the data in intervals from 1 to 40 nights. Indeed, a year later, the High Accuracy Radial velocity Planet Searcher (HARPS) team published the smallest-velocity signal ever detected: a planet candidate that orbits $\alpha$ Centauri B (Dumusque et al., 2012) with a velocity amplitude $\mathrm{K}=0.51 \mathrm{~m} \mathrm{~s}^{-1}$, planet mass $\mathrm{M} \sin \mathrm{i}=1.13 \mathrm{M}_{\oplus}$, and an orbital period of $3.24 \mathrm{~d}$. This detection required 469 Doppler measurements obtained over seven years and fit for several time-variable stellar noise signals. Thus, the number of observations required to solve for the five-parameter Keplerian model increases exponentially with decreasing velocity amplitude.

\subsection{The Future of Doppler Detections}

It is worth pondering whether improved instruments with higher resolution, higher sampling, greater stability, and more precise wavelength calibration will ultimately be able to detect analogs of Earth with $0.1-\mathrm{m} \mathrm{s}^{-1}$ velocity amplitudes. An extreme precision spectrometer will have stringent environmental requirements to control temperature, pressure, and vibrations. The dual requirements of high resolution and high $\mathrm{S} / \mathrm{N}$ lead to the need for moderate- to large-aperture telescopes (Strassmeier et al., 2008; Spanò et al., 2012). The coupling of light into the instrument must be exquisitely stable. This can be achieved with a double fiber scrambler (Hunter and Ramsey, 1992) where the near field of the input fiber is mapped to the far field of the output fiber, providing a high level of scrambling in both the radial and azimuthal directions. At some cost to throughput, the double fiber scrambler stabilizes variations in the spectral line spread function (sometimes called a point

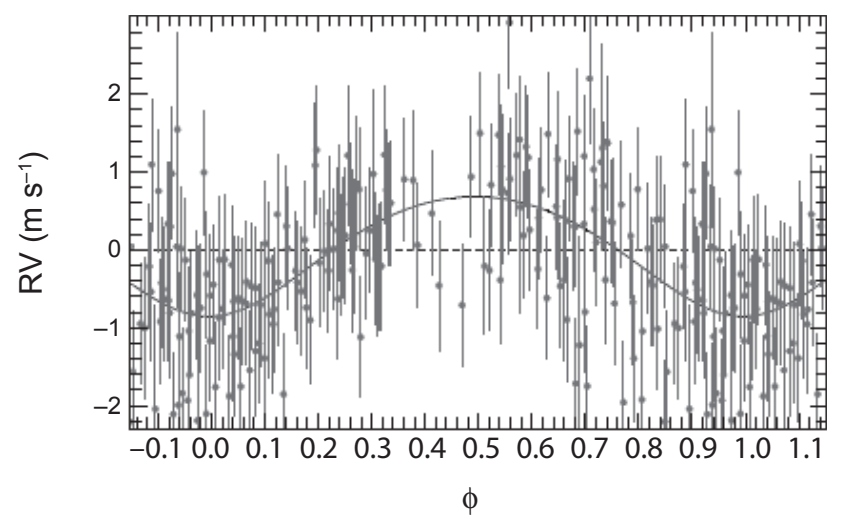

Fig. 2. The phase-folded data for the detection of a planet orbiting HD 85512. From Fig. 13 of Pepe et al. (2011).

spread function) and produces a series of spectra that are uniform except for photon noise. Although the fibers provide superior illumination of the spectrometer optics, some additional care in the instrument design phase is required to provide excellent flat fielding and sky subtraction. The list of challenges to extreme instrumental precision also includes the optical CCD detectors, with intrapixel quantum efficiency variations, tiny variations in pixel sizes, charge diffusion, and the need for precise controller software to perfectly clock readout of the detector.

In addition to the instrumental precision, another challenge to high Doppler precision is the star itself. Stellar activity, including star spots, p-mode oscillations, and variable granulation, are tied to changes in the strength of stellar magnetic fields. These stellar noise sources are sometimes called stellar jitter and can produce line profile variations that skew the center of mass for a spectral line in a way that is (mis)interpreted by a Doppler code as a velocity change in the star. Although stellar noise signals are subtle, they affect the spectrum in a different way than dynamical velocities. The stellar noise typically has a color dependence and an asymmetric velocity component. In order to reach significantly higher accuracy in velocity measurements, it is likely that we will need to identify and model or decorrelate the stellar noise.

\section{INFRARED SPECTROSCOPY}

\subsection{Doppler Radial Velocities in the Near Infrared}

The high fraction of Earth-sized planets estimated to orbit in the habitable zones (HZs) of M dwarfs (Dressing and Charbonneau, 2013; Kopparapu, 2013; Bonfils et al., 2013) makes the low mass stars very attractive targets for Doppler radial velocity (RV) surveys. The lower stellar mass of the M dwarfs, as well as the short orbital periods of HZ planets, increases the amplitude of the Doppler wobble (and the ease of its detectability) caused by such a terrestrial-mass planet. However, nearly all the stars in current optical RV surveys 
are earlier in spectral type than $\sim \mathrm{M} 5$, since later spectral types are difficult targets even on large telescopes due to their intrinsic faintness in the optical: They emit most of their flux in the red optical and near-infrared (NIR) between 0.8 and $1.8 \mu \mathrm{m}$ (the NIR Y, J, and $\mathrm{H}$ bands are $0.98-1.1 \mu \mathrm{m}$, $1.1-1.4 \mu \mathrm{m}$, and $1.45-1.8 \mu \mathrm{m})$. However, it is the low-mass late-type $\mathrm{M}$ stars, which are the least luminous, where the velocity amplitude of a terrestrial planet in the habitable zone is highest, making them very desirable targets. Since the flux distribution from $M$ stars peaks sharply in the NIR, stable high-resolution NIR spectrographs capable of delivering high RV precision can observe several hundred of the nearest $\mathrm{M}$ dwarfs to examine their planet population.

3.1.1. Fiber-fed near-infrared high-resolution spectrographs. A number of new fiber-fed stabilized spectrographs are now being designed and built for such a purpose: the Habitable Zone Planet finder (Mahadevan et al., 2012) for the 10-m Hobby Eberly Telescope, the Calar Alto high-Resolution search for $\mathrm{M}$ dwarfs with Exo-earths with Near-infrared and Visible Echelle Spectrographs (CARMENES) (Quirrenbach et al., 2012) for the 3.6-m Calar Alto Telescope, and SpectroPolarimètre Infra-Rouge (SPIRou) (Santerne et al., 2013) being considered for the Canada-France-Hawaii Telescope (CFHT). The instrumental challenges in the NIR, compared to the optical, are calibration, stable cold operating temperatures of the instrument, and the need to use NIR detectors. The calibration issues seem tractable (see below). Detection of light beyond $1 \mu \mathrm{m}$ required the use of NIR sensitive detectors like the Hawaii-2 (or 4) RG HgCdTe detectors. These devices are fundamentally different than charge-coupled devices (CCDs) and exhibit effects such as interpixel capacitance and much greater persistence. Initial concerns about the ability to perform precision RV measurements with these devices has largely been retired with laboratory (Ramsey et al., 2008) and on-sky demonstrations (Ycas et al., 2012b) with a Pathfinder spectrograph, although careful attention to ameliorating these effects is still necessary to achieve high $\mathrm{RV}$ precision. This upcoming generation of spectrographs, being built to deliver $1-3-\mathrm{m} \mathrm{s}^{-1} \mathrm{RV}$ precision in the NIR, will also be able to confirm many of the planets detected with the Transiting Exoplanet Survey Satellite (TESS) and Gaia around low-mass stars. Near-infrared spectroscopy is also an essential tool to be able to discriminate between giant planets and stellar activity in the search for planets around young active stars (Mahmud et al., 2011).

3.1.2. Calibration sources. Unlike iodine in the optical, no single known gas cell simultaneously covers large parts of the NIR z, Y, J, and $\mathrm{H}$ bands. Thorium-argon lamps, which are so successfully used in the optical, have very few thorium emission lines in the NIR, making them unsuitable as the calibrator of choice in this wavelength regime. Uranium has been shown to provide a significant increase in the number of lines available for precision wavelength calibration in the NIR. New linelists have been published for uranium lamps (Redman et al., 2011, 2012) and these lamps are now in use in existing and newly commissioned
NIR spectrographs. Laser frequency combs, which offer the prospects of very high precision and accuracy in wavelength calibration, have also been demonstrated with astronomical spectrographs in the NIR (Ycas et al., 2012b), with filtering making them suitable for an astronomical spectrograph. A generation of combs spanning the entire $\mathrm{z}-\mathrm{H}$-band region has also been demonstrated in the laboratory (Ycas et al., 2012a). Continuing development efforts are aimed at effectively integrating these combs as calibration sources for M-dwarf Doppler surveys with stabilized NIR spectrographs. Single mode fiber-based Fabry-Pérot cavities fed by supercontinuum light sources have also been demonstrated by Halverson et al. (2012). To most astronomical spectrographs the output from these devices looks similar to that of a laser comb, although the frequency of the emission peaks is not known innately to high precision. Such inexpensive and rugged devices may soon be available for most NIR spectrographs, with the superior (and more expensive) laser combs being reserved for the most stable instruments on the larger facilities. While much work remains to be done to refine these calibration sources, the calibration issues in the NIR largely seem to be within reach.

3.1.3. Single-mode fiber-fed spectrographs. The advent of high strehl ratio adaptive optics (AO) systems at most large telescopes makes it possible to seriously consider using a single-mode optical fiber (SMF) to couple the light from the focal plane of the telescope to a spectrograph. Working close to the diffraction limit enables such SMFfed spectrographs to be very compact while simultaneously capable of providing spectral resolution comparable or superior to natural-seeing spectrographs. A number of groups are pursuing technology development relating to these goals (Ghasempour et al., 2012; Schwab et al., 2012; Crepp, 2013). The SMFs provide theoretically perfect scrambling of the input point spread function (PSF), further aiding in the possibility of very-high-precision and compact Doppler spectrometers emerging from such development paths. While subtleties relating to polarization state and its impact on velocity precision remain to be solved, many of the calibration sources discussed above are innately adaptable to use with SMF fiber-fed spectrographs. Since the efficiency of these systems depends steeply on the level of AO correction, it is likely that Doppler RV searches targeting the red optical and NIR wavelengths will benefit the most.

\subsection{Spectroscopic Detection of Planetary Companions}

Direct spectroscopic detection of the orbit of nontransiting planets has finally yielded successful results this decade. While the traditional Doppler technique relies on detecting the radial velocity of the star only, the direct spectroscopic detection technique relies on observing the star-planet system in the NIR or thermal IR (where the planet-to-star flux ratio is more favorable than the optical) and obtaining high-resolution, very high $\mathrm{S} / \mathrm{N}$ spectra to be able to spectroscopically measure the radial velocity of both the star and the planet in a manner analogous to the detection 
of a spectroscopic binary (SB2). The radial velocity observations directly yield the mass ratio of the star-planet system. If the stellar mass is known (or estimated well), the planet mass can be determined with no sin i ambiguity despite the fact that these are not transiting systems. The spectroscopic signature of planets orbiting Tau Boo, 51 Peg, and HD 189733 have recently been detected using the CRyogenic high-resolution InfraRed Echelle Spectrograph (CRIRES) instrument on the Very Large Telescope (VLT) (Brogi et al., 2012, 2013; de Kok et al., 2013; Rodler et al., 2012), and efforts are ongoing by multiple groups to detect other systems using the Near Infrared Echelle Spectrograph (NIRSPEC) instrument at Keck Observatory (Lockwood et al., 2014). The very high $\mathrm{S} / \mathrm{N}$ required of this technique limits it to the brighter planet hosts and to relatively closein planets, but yields information about mass and planetary atmospheres that would be difficult to determine otherwise for the nontransiting planets. Such techniques complement the transit detection efforts underway and will increase in sensitivity with telescope aperture, better infrared detectors, and more sophisticated analysis techniques. While we have focused primarily on planet-detection techniques in this review article, high-resolution NIR spectroscopy using large future groundbased telescopes may also be able to detect astrobiologically interesting molecules (e.g., $\mathrm{O}_{2}$ ) around Earth-analogs orbiting M dwarfs (Snellen et al., 2013).

\section{DOPPLER MEASUREMENTS FROM SPACE}

Although there are no current plans to build high-resolution spectrometers for space missions, this environment might offer some advantages for extreme-precision Doppler spectroscopy if the instrument would be in a stable thermal and pressure environment. Without blurring from Earth's atmosphere, the PSF would be very stable and the image size could be small, making it intrinsically easier to obtain high resolution with an extremely compact instrument. Furthermore, the effect of sky subtraction and telluric contamination are currently difficult problems to solve with groundbased instruments, and these issues are eliminated with spacebased instruments.

\section{TRANSIT DETECTIONS}

At the time of the publication of the Protostars and Planets IV (PPIV) volume in 2000 (Mannings et al., 2000), the first transiting extrasolar planet - HD 209458b - had just been found (Henry et al., 2000; Charbonneau et al., 2000). That momentous announcement, however, was too late for the conference volume, and $P P I V$ 's single chapter on planet detection was devoted to 14 planets detected by Doppler velocity monitoring, of which only 8 were known prior to the June 1998 meeting. Progress, however, was rapid. In 2007, when the Protostars and Planets $V$ volume was published (Reipurth et al., 2007), nearly 200 planets had been found with Doppler radial velocities, and 9 transiting planets were known at that time (Charbonneau et al., 2007).
In the past several years, the field of transit detection has come dramatically into its own. A number of long-running groundbased projects, notably the SuperWASP (Collier Cameron et al., 2007) and HATNet surveys (Bakos et al., 2007), have amassed the discovery of dozens of transiting planets with high-quality light curves in concert with accurate masses determined via precision Doppler velocity measurements. Thousands of additional transiting planetary candidates have been observed from space. Transit timing variations (Agol et al., 2005; Holman and Murray, 2005) have progressed from a theoretical exercise to a practiced technique. The Spitzer Space Telescope (along with the Hubble Space Telescope and groundbased assets) has been employed to characterize the atmospheres of dozens of transiting extrasolar planets (Seager and Deming, 2010). An entirely new, and astonishingly populous, class of transiting planets in the mass range $\mathrm{R}_{\oplus}<\mathrm{R}_{\mathrm{P}}<4 \mathrm{R}_{\oplus}$ has been discovered and probed (Batalha et al., 2013). Certainly, with each new iteration of the Protostars and Planets series, the previous edition looks hopelessly quaint and out of date. Is seems certain that progress will ensure that this continues to be the case.

\subsection{The Era of Spacebased Transit Discovery}

Two space missions, Kepler (Borucki et al., 2010) and Convection, Rotation and planetary Transits (CoRoT) (Barge et al., 2008), have both exhibited excellent productivity, and a third mission, Microvariability and Oscillations of Stars (MOST), has provided photometric transit discoveries of several previously known planets (Winn et al., 2011; Dragomir et al., 2013). Indeed, Fig. 1 shows that during the past six years, transiting planets have come to dominate the roster of new discoveries. Doppler velocimetry, which was overwhelmingly the most productive discovery method through 2006, is rapidly transitioning from a general survey mode to an intensive focus on low-mass planets orbiting very nearby stars (Mayor et al., 2011) and to the characterization of planets discovered in transit via photometry.

The Kepler mission, in particular, has been completely transformative, having generated, at last rapidly evolving count, over 100 planets with mass determinations, as well as hundreds of examples of multiple transiting planets orbiting a single host star, many of which are in highly co-planar, surprisingly crowded systems (Lissauer et al., 2011b). Taken in aggregate, the Kepler candidates indicate that planets with masses $\mathrm{M}_{\mathrm{P}}<30 \mathrm{M}_{\oplus}$ and orbital periods $\mathrm{P}<100 \mathrm{~d}$ are effectively ubiquitous (Batalha et al., 2011), and as shown in Fig. 3, the distribution of mass ratios and periods of these candidate planets are, in many cases, curiously reminiscent of the regular satellites of the jovian planets within our own solar system.

The CoRoT satellite ceased active data gathering in late 2012, having substantially exceeded its three-year design life. In spring of 2013, just after the end of its nominal mission period, the Kepler satellite experienced a failure of a second reaction wheel, which brought its high- 


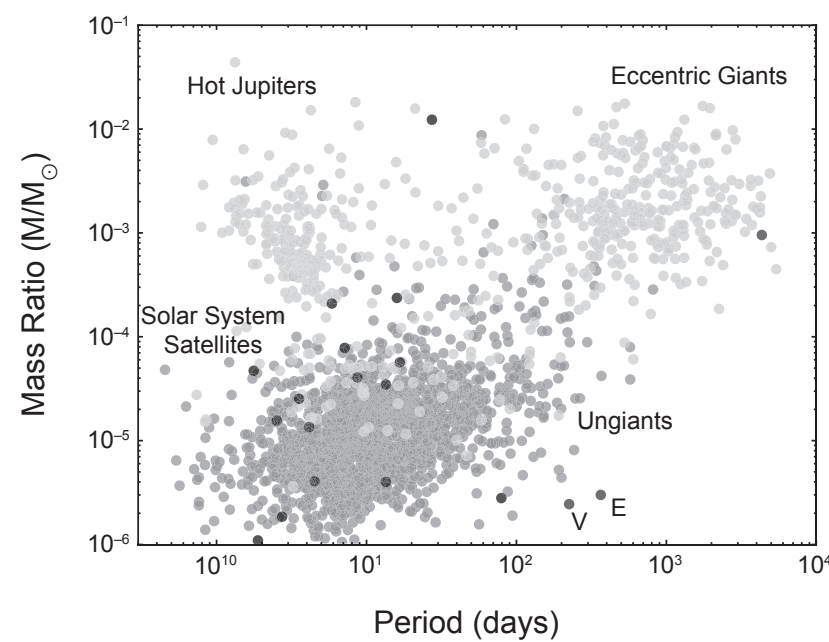

Fig. 3. Medium-gray circles: $\log _{10}\left(M_{\text {satellite }} / M_{\text {primary }}\right)$ and $\log _{10}(P)$ for 634 planets securely detected by the radial velocity method (either with or without photometric transits). Light-gray circles: $\log _{10}\left(M_{\text {satellite }} / M_{\text {primary }}\right)$ and $\log _{10}(P)$ for the regular satellites of the jovian planets in the solar system. Dark-gray circles: $\log _{10}\left(M_{\text {satellite }} / M_{\text {primary }}\right)$ and $\log _{10}(P)$ for 1501 Kepler candidates and objects of interest in which multiple transiting candidate planets are associated with a single primary. Radii for these candidate planets, as reported in Batalha et al. (2013), are converted to masses assuming $\mathrm{M} / \mathrm{M}_{\oplus}=\left(\mathrm{R} / \mathrm{R}_{\oplus}\right)^{2.06}$ (Lissauer et al., 2011a), which is obtained by fitting the masses and radii of the solar system planets bounded in mass by Venus and Saturn. Data are from www. exoplanets.org as of August 15, 2013.

precision photometric monitoring program to a premature halt. The four years of Kepler data in hand, however, are well-curated, fully public, and are still far from being fully exploited; it is not unreasonable to expect that they will yield additional insight that is equivalent to what has already been gained from the mission to date. Jenkins et al. (2010) describe the fiducial Kepler pipeline; steady improvements to the analysis procedures therein have led to large successive increases in the number of planet candidates detected per star (Batalha et al., 2013).

The loss of the Kepler and CoRoT spacecraft has been tempered by the recent approvals of two new space missions. In the spring of 2013, NASA announced selection of the TESS mission for its Small Explorer Program. The TESS mission is currently scheduled for a 2017 launch. It will employ an all-sky strategy to locate transiting planets with periods of weeks to months, and sizes down to $R_{p} \sim 1 R_{\oplus}$ (for small parent stars) among a sample of $5 \times 10^{5}$ stars brighter than $\mathrm{V}=12$, including $\sim 1000$ red dwarfs (Ricker et al., 2010). TESS is designed to take advantage of the fact that the most heavily studied, and therefore the most scientifically valuable, transiting planets in a given category (hot Jupiters, extremely inflated planets, sub-Neptune-sized planets, etc.) orbit the brightest available parent stars. To date, many of these "fiducial" worlds, such as HD 209458b, HD 149026b,
HD $189733 \mathrm{~b}$, and Gliese 436b, have been discovered to transit by photometrically monitoring known Doppler-wobble stars during the time windows when transits are predicted to occur. By surveying all the bright stars, TESS will systematize the discovery of the optimal transiting example planets within every physical category. The CHaracterising ExOPlanet Satellite (CHEOPS) is also scheduled for launch in 2017 (Broeg et al., 2013). It will complement TESS by selectively and intensively searching for transits by candidate planets in the $\mathrm{R}_{\oplus}<\mathrm{R}_{\mathrm{p}}<4 \mathrm{R}_{\oplus}$ size range during time windows that have been identified by high-precision Doppler monitoring of the parent stars. It will also perform follow-up observations of interesting TESS candidates.

\subsection{Transit Detection}

The a priori probability that a given planet can be observed in transit is a function of the planetary orbit, and the planetary and stellar radii

$$
\mathcal{P}_{\text {tr }}=0.0045\left(\frac{\mathrm{AU}}{\mathrm{a}}\right)\left(\frac{\mathrm{R}_{\star}+\mathrm{R}_{\mathrm{p}}}{\mathrm{R}_{\odot}}\right)\left[\frac{1+\mathrm{e} \cos (\pi / 2-\omega)}{1-\mathrm{e}^{2}}\right]
$$

where $\omega$ is the angle at which orbital periastron occurs, such that $\omega=90^{\circ}$ indicates transit, and e is the orbital eccentricity. A typical hot Jupiter with $\mathrm{R}_{\mathrm{p}} \geq \mathrm{R}_{\text {Jup }}$ and $\mathrm{P} \sim 3 \mathrm{~d}$, orbiting a solar-type star, has a $\tau \sim 3 \mathrm{hr}$ transit duration, a photometric transit depth, $\mathrm{d} \sim 1 \%$, and $\mathcal{P} \sim 10 \%$. Planets belonging to the ubiquitous super-Earth-sub-Neptune population identified by Kepler (i.e., the gray points in Fig. 3) are typified by $\mathcal{P} \sim 2.5 \%, \mathrm{~d} \sim 0.1 \%$, and $\tau \sim 6 \mathrm{hr}$, whereas Earth-sized planets in an Earth-like orbits around solar-type stars present a challenging combination of $\mathcal{P} \sim 0.5 \%, \mathrm{~d} \sim$ $0.01 \%$, and $\tau \sim 15 \mathrm{hr}$.

Effective transit search strategies seek the optimal tradeoff between cost, sky coverage, photometric precision, and the median apparent brightness of the stars under observation. For nearly a decade, the community as a whole struggled to implement genuinely productive surveys. For an interesting summary of the early disconnect between expectations and reality, see Horne (2003). Starting in the mid-2000s, however, a number of projects began to produce transiting planets (Konacki et al., 2003; Alonso et al., 2004; McCullough et al., 2006), and there are now a range of successful operating surveys. For example, the ongoing Kelt-North project, which has discovered four planets to date (Collins et al., 2013), targets very bright $8<\mathrm{V}<10$ stars throughout a set of $26^{\circ} \times 26^{\circ}$ fields that make up $\sim 12 \%$ of the full sky. Among nearly 50,000 stars in this survey, 3822 targets have RMS photometric precision better than $1 \%$ (for 150-s exposures). A large majority of the known transit-bearing stars, however, are fainter than Kelt's faint limit near $\mathrm{V} \sim 10$. The $10<\mathrm{V}<12$ regime has been repeatedly demonstrated to provide good prospects for Doppler follow-up and detailed physical characterization, along with a large number of actual transiting planets. In 
this stellar brightness regime, surveys such as HATNet and SuperWASP have led the way. For instance, HAT-South (Bakos et al., 2013), a globally networked extension of the long-running HATNet project, monitors $8.2^{\circ} \times 8.2^{\circ}$ fields and reaches 6 millimagnitude (mmag) photometric precision at a four-minute cadence for the brightest nonsaturated stars at $r \sim 10.5$. SuperWASP's characteristics are roughly similar, and to date, it has been the most productive groundbased transit search program.

To date, the highest-precision groundbased exoplanetary photometry has been obtained with orthogonal phase transfer arrays trained on single, carefully preselected high-value target stars. Using this technique, Johnson et al. (2009) obtained 0.47-mmag photometry at 80-s cadency for WASP-10 ( $\mathrm{V}=12.7)$. By comparison, with its spaceborne vantage, Kepler obtained a median photometric precision of 29 ppm with 6.5-hour cadence on $\mathrm{V}=12$ stars. This is $\sim 2 \times$ better than the best special-purpose groundbased photometry, and $\sim 20 \times$ better than the leading groundbased discovery surveys.

Astrophysical false positives present a serious challenge for wide-field surveys in general and for Kepler in particular, where a majority of the candidate planets lie effectively out of reach of Doppler characterization and confirmation (Morton and Johnson, 2011). Stars at the bottom of the main sequence overlap in size with giant planets (Chabrier and Baraffe, 2000) and thus present near-identical transit signatures to those of giant planets. Grazing eclipsing binaries can also provide a source of significant confusion for low S/N light curves (Konacki et al., 2003).

Within the Kepler field, pixel "blends" constitute a major channel for false alarms. These occur when an eclipsing binary, either physically related or unrelated, shares line of sight with the target star. Photometry alone can be used to identify many such occurrences (Batalha et al., 2010), whereas in other cases, statistical modeling of the likelihood of blend scenarios (Torres et al., 2004; Fressin et al., 2013) can establish convincingly low false alarm probabilities. High-profile examples of confirmation by statistical validation include the $\mathrm{R}=2.2 \mathrm{R}_{\oplus}$ terrestrial candidate planet Kepler 10c by (Fressin et al., 2011), as well as the planets in the Kepler 62 system (Borucki et al., 2013). False alarm probabilities are inferred to be dramatically lower for cases where multiple candidate planets transit the same star. Among the gray points in Fig. 3 there is very likely only a relatively small admixture of false alarms.

\subsection{Results and Implications}

Aside from the sheer increase in the number of transiting planets that are known, the string of transit discoveries over the past six years have been of fundamentally novel importance. In particular, transit detections have enabled the study of both planets and planetary system architectures for which there are no solar system analogs. A brief tally of significant events logged in order of discovery year might include (1) Gliese 436 b (Gillon et al., 2007), the first tran- siting Neptune-sized planet and the first planet to transit a low-mass star; (2) HD 17156b, the first transiting planet with a large orbital eccentricity $(\mathrm{e}=0.69)$ and an orbital period $(\mathrm{P}=21 \mathrm{~d})$ that is substantially larger than the $2 \mathrm{~d}<\mathrm{P}<$ $5 \mathrm{~d}$ range occupied by a typical hot Jupiter (Barbieri et al., 2007); (3) CoRoT 7 b (Léger et al., 2009) and Gliese 1214b (Charbonneau et al., 2009), the first transiting planets with masses in the so-called "super-Earth" regime $1 \mathrm{M}_{\oplus}<\mathrm{M}<$ $10 \mathrm{M}_{\oplus}$; (4) Kepler 9b and 9c (Holman et al., 2010), the first planetary system to show tangible transit timing variations, as well as the first case of transiting planets executing a low-order mean-motion resonance; (5) Kepler 22b, the first transiting planet with a size and an orbital period that could potentially harbor an Earth-like environment (Borucki et al., 2012); and (6) the Kepler 62 system (Borucki et al., 2013), which hosts at least five transiting planets orbiting a K2V primary. The outer two members, Planet "e" with $\mathrm{P}=122 \mathrm{~d}$ and Planet "f" with $\mathrm{P}=267 \mathrm{~d}$, both have $1.25 \mathrm{R}_{\oplus}<\mathrm{R}_{\mathrm{p}}<$ $2 \mathrm{R}_{\oplus}$, and receive $\mathrm{S}=1.2 \pm 0.2 \mathrm{~S}_{\odot}$ and $\mathrm{S}=0.4 \pm 0.05 \mathrm{~S}_{\odot}$ of Earth's solar flux respectively.

Bulk densities are measured for transiting planets with parent stars that are bright enough and chromospherically quiet enough to support Doppler measurement of $\mathrm{M}_{\mathrm{P}} \sin \mathrm{i}$, and can also be obtained by modeling transit timing variations (Fabrycky et al., 2012; Lithwick et al., 2012). More than 100 planetary densities (mostly for hot Jupiters) have been securely measured. These are plotted in Fig. 4, which hints at the broad outlines of an overall distribution. Figure 4 is anticipated to undergo rapid improvement over the next several years as more Kepler candidates receive mass determinations. It appears likely, however, that there exists a very broad range of planetary radii at every mass. For

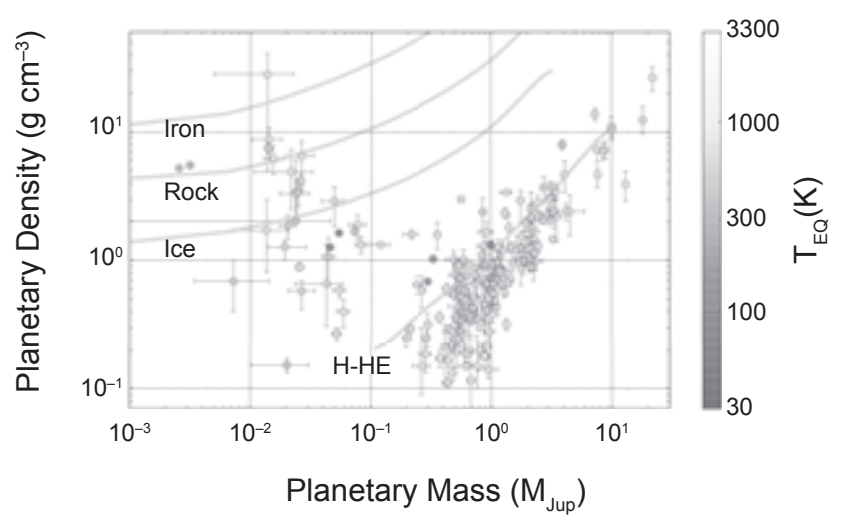

Fig. 4. Density-mass diagram for planets with well-determined masses and radii. Planets are color-coded by the equilibrium temperature, $T_{\text {eq }}=\left(R_{\star}^{1 / 2} T_{\star}\right) /\left((2 a)^{1 / 2}\left(1-e^{2}\right)^{1 / 8}\right)$ that they would have if they were zero-albedo blackbodies reradiating from the full planetary surface area. The solar system planets more massive than Mars are included in the plotted aggregate. Gray lines show expected $\rho\left(M_{P}\right)$ for planetary models of pure hydrogen-helium, pure water, pure silicate, and pure iron compositions. Planetary data are from $w w w$. exoplanets.org as of August 15, 2013. 
example, to within errors, planets with $\mathrm{M}_{\mathrm{P}} \sim 6 \mathrm{M}_{\oplus}$ appear to range in radius by a factor of at least 3 . While a substantial number of short-period giant planets are inflated by unknown energy source(s) (Batygin and Stevenson, 2010), compositional variations are at least capable of explaining the observed range of radii for planets with $\mathrm{M}_{\mathrm{P}}<0.2 \mathrm{M}_{\text {Jup }}$ (Fortney et al., 2007). The mass-density distribution (and by extension, the composition distribution) of extrasolar planets as a function of stellocentric distance is an important outcome of the planet-formation process. It is still entirely unclear whether planets with $\mathrm{P}<100 \mathrm{~d}$ that have no solar system analogs are the product of migration processes (Ida and Lin, 2004) or of in situ formation (Chiang and Laughlin, 2013). More high-quality measurements of transiting planets will be required to resolve the puzzle.

The large number of candidate multiple transiting planet systems indicate that co-planar architectures are the rule for planets with $\mathrm{P}<100 \mathrm{~d}$ in the size range of $\mathrm{R}_{\mathrm{p}} \sim 1.5-6 \mathrm{R}_{\oplus}$ (Moorhead et al., 2011). The inclination dispersion of most candidate systems with two or more transiting planets appears to have a median between $1^{\circ}$ and $3^{\circ}$. Candidate planets in multiple-transit systems, furthermore, are invariably in dynamically stable configurations when imbued with reasonable mass-radius relations (Lissauer et al., 2011a). Nature has therefore produced a galactic planetary census that is extraordinarily well suited to detection and characterization via the transit method. The advent of the new space missions, in concert with the James Webb Space Telescope's (JWST) potential for atmospheric characterization of low-mass planets (Deming et al., 2009), indicate that transits will remain at the forefront for decades to come.

Finally, transit detection is unique in that it democratizes access to cutting-edge research in exoplanetary science. Nearly all the highly cited groundbased discoveries have been made with small telescopes of aperture $d<1 \mathrm{~m}$. Amateur observers were co-discoverers of the important transits by HD 17156b (Barbieri et al., 2007) and HD 80606b (Garcia-Melendo and McCullough, 2009), and citizen scientists have discovered several planets to date in the Kepler data under the auspices of the Planet Hunters project (Fischer et al., 2012; Lintott et al., 2013; Schwamb et al., 2013).

\section{DIRECT IMAGING TECHNIQUES}

The field of exoplanets is almost unique in astronomical science in that the subjects are almost all studied indirectly, through their effects on more visible objects, rather than being imaged themselves. The study of the dominant constituents of the universe (dark energy and dark matter) through their gravitational effects is of course another example. Direct imaging of the spatially resolved planet is a powerful complement to the other techniques described in this chapter. It is primarily sensitive to planets in wide orbits a $>5 \mathrm{AU}$, and since photons from the planets are recorded directly, the planets are amenable to spectroscopic or photometric characterization. However, direct detection also represents a staggering technical challenge. If a twin to our solar system were located at a distance of 10 pc from Earth, the brightest planet would have only $\sim 10^{-9}$ the flux of the parent star, at an angular separation of 0.5 arcsec.

In spite of this challenge, the field has produced a small number of spectacular successes: the images and spectra of massive $\left(>1000 \mathrm{M}_{\oplus}\right)$ young self-luminous planets. The advent of the first dedicated exoplanet imaging systems should lead to rapid progress and surveys with statistical power comparable to groundbased Doppler or transit programs. In the next decade, spacebased coronagraphs will bring mature planetary systems into reach, and some day, a dedicated exoplanet telescope may produce an image of an Earth analog orbiting a nearby star.

\subsection{Limitations to High-Contrast Imaging}

The greatest challenge in direct imaging is separating the light of the planet from residual scattered light from the parent star. This can be done both optically - removing the starlight before it reaches the science detector - and in post-processing, using a feature that distinguishes starlight from planetary light.

6.1.1. High-contrast point spread function, coronagraphs, and adaptive optics. Even in the absence of aberrations, the images created by a telescope will contain features that will swamp any conceivable planet signal. The PSF, as the name implies, is the response of the telescope to an unresolved point source. In the case of an unaberrated telescope, the PSF is the magnitude squared of the Fourier transform of the telescope aperture function. For an unobscured circular aperture, the diffraction pattern is the distinctive Airy rings. (The one-dimensional equivalent would represent the telescope as a top hat function, whose Fourier transform is a sync, giving a central peak and oscillating sidelobes.) More complex apertures will have more complex diffraction patterns.

Removing this diffraction pattern is the task of a coronagraph. Originally developed by Lyot (1939) to allow small telescopes to study the coronae of the Sun, chronographs employ optical trickery to remove the light from an onaxis star while allowing some of the flux from the off-axis planet to remain. A wide variety of approches have been developed (Guyon et al., 2006), far too many to enumerate here, although they can be divided into broad families. The classical Lyot coronagraph blocks the on-axis source with a focal plane mask, followed by a pupil-plane Lyot mask that blocks the light diffracted by the focal plane (Lyot, 1939; Sivaramakrishnan et al., 2001). Apodizers operate by modifying the transmission of the telescope so that the Fourier transform has substantially less power in the sidelobes; a nonphysical example would be a telescope whose transmission was a smoothly varying Gaussian, which would result in a purely Gaussian PSF. In more practical designs, apodization is implemented through binary "shaped pupil" masks (Kasdin et al., 2003) that sharply reduce diffraction over a target region at a significant cost in throughput. Hybrid Lyot approaches use pupil-plane apodization (Soummer et al., 2011) or complicated focal-plane masks (Kuchner 
and Traub, 2002) to boost the performance of the classic Lyot. Phase-induced amplitude apodization uses complex mirrors to create the tapered beam needed to suppress diffraction without a loss in throughput (Guyon et al., 2005). A particularly promising new technique creates an optical vortex in the focal plane (Nersisyan et al., 2013), removing the diffracted light almost perfectly for an on-axis source in a unobscured aperture. Many more complex coronagraphs exist (see Guyon et al., 2006, for discussion). Typically, the best coronagraphs remove diffraction down to the level of $10^{-10}$ at separations greater than the inner working angle (IWA), typically $2-4 \lambda / \mathrm{D}$.

Light is also scattered by optical imperfections wavefront errors induced by the telescope, camera, or atmospheric turbulence. Even with a perfect coronagraph, atmospheric turbulence, which typically is many waves of phase aberration, produces a PSF that completely overwhelms any planetary signal. Even in the absence of atmospheric turbulence, small wavefront errors from, e.g., polishing marks will still scatter starlight. These can be partially corrected through adaptive optics — using a deformable mirror (DM), controlled by some estimate of the wavefront, to correct the phase of the incoming light. In the case of small phase errors, a Fourier relationship similar to that for diffraction exists between the wavefront and PSF (see Perrin et al., 2003, and Guyon et al., 2006, for discussion and examples). A useful figure of merit for adaptive optics correction is the Strehl ratio, defined as the ratio of the peak intensity of the measured PSF to the theoretical PSF for an equivalent unaberrated telescope. With current-generation adaptive optics systems, Strehl ratios of $0.4-0.8$ are common in the $\mathrm{K}$ band - meaning that $60-80 \%$ of the scattered light remains uncorrected.

The halo of light scattered by wavefront errors is particularly troublesome because it does not form a smooth background, but is broken up into a pattern of speckles. In monochromatic light these speckles resemble the diffraction-limited PSF of the telescope, and hence are easily confused with the signal from a planet. As a result, high-contrast images are usually nowhere near the Poisson limit of photon noise but instead limited by these speckles. Uncorrected atmospheric turbulence produces a halo of speckles that rapidly evolve; static or quasistatic wavefront errors, such as adaptive optics miscalibrations, produce slowly evolving speckles that mask planetary signals.

6.1.2. Post-processing. These speckle patterns can be partially mitigated in post-processing. Such PSF subtraction requires two components. First, there must be some distinction between a planetary signal and the speckle pattern - some diversity. Examples include wavelength diversity, where the wavelength dependence of the speckle pattern differs from that of the planet; rotational diversity, in which the telescope (and associated speckle pattern) rotates with respect to the planet/star combination (Marois et al., 2006); or observations of a completely different target star. Such reference PSFs will never be a perfect match, as the PSF evolves with time, temperature, star brightness, and wavelength. The second component needed for effective PSF subtraction is an algorithm that can construct the "best" PSF out of a range of possibilities. With a suitable library of PSFs, least-squares fitting (Lafrenière et al., 2007a) or principal components analysis can assemble synthetic PSFs and enhance sensitivity to planets by a factor of $10-100$.

\subsection{Imaging of Self-Luminous Planets}

With these techniques applied to current-generation systems, planets with brightness $\sim 10^{-5}$ can be seen at angular separations of $\sim 1.0 \mathrm{arcsec}$. This is far from the level of sensitivity needed to see mature Jupiter-like planets. Fortunately, planets are available that are much easier targets. When a planet forms, significant gravitational potential energy is available. Depending on the details of initial conditions, a newly formed giant planet may have an effective temperature of 1000-2000 K (Marley et al., 2007) and a luminosity of $10^{-5}-10^{-6} \mathrm{~L}_{\odot}$ (Fig. 5). As with the brown dwarfs, a large fraction of this energy could be released in the NIR, bringing the planet into the detectable range. Such planets remain detectable for tens of millions of years. Several surveys have targeted young stars in the solar neighborhood for exoplanet detection (Liu et al., 2010; Lafrenière et al., 2007b; Chauvin et al., 2010), benefitting from the identification of nearby young associations composed of stars with ages 8-50 million years (m.y.) (Zuckerman and Song, 2004). Most of these surveys have produced only nondetections, with upper limits on the number of giant planets as a function of semimajor axis that exclude large numbers of very-wide-orbit (50 AU) planets.

A handful of spectacular successes have been obtained. One of the first detections was a $5 \mathrm{M}_{\text {Jup }}$ object that was orbiting not a star but a young brown dwarf, 2M1207B (Chauvin et al., 2004). A spectacular example of planetary companions to a main-sequence star is the HR 8799 multiplanet system (Fig. 6). This consists of four objects near a young F0V star, orbiting in counterclockwise directions. The object's luminosities are well constrained by broadband photometry (Marois et al., 2008; Currie et al., 2011). Estimates of the planetary mass depend on knowledge of the stellar age - thought to be 30 m.y. (Marois et al., 2010; Baines et al., 2012) - and initial conditions; for "hot start" planets the masses are 3-7× that of Jupiter. Multi-planet gravitational interactions provide a further constraint on the mass (Marois et al., 2010; Fabrycky and Murray-Clay, 2010), excluding massive brown dwarf companions. Other notable examples of directly imaged exoplanets include the very young object 1RXS J1609b (Lafrenière et al., 2010), the cool planet candidate GJ 504B (Kuzuhara et al., 2013), and the planet responsible for clearing the gap inside the $\beta$ Pictoris disk (Lagrange et al., 2010). A candidate optical HST image of an exoplanet was reported orbiting Fomalhaut (Kalas et al., 2008), but very blue colors and a belt-crossing orbit (Kalas et al., 2013) indicate that what is seen is likely light scattered by a debris cloud or disk (that may still be associated with a planet). 


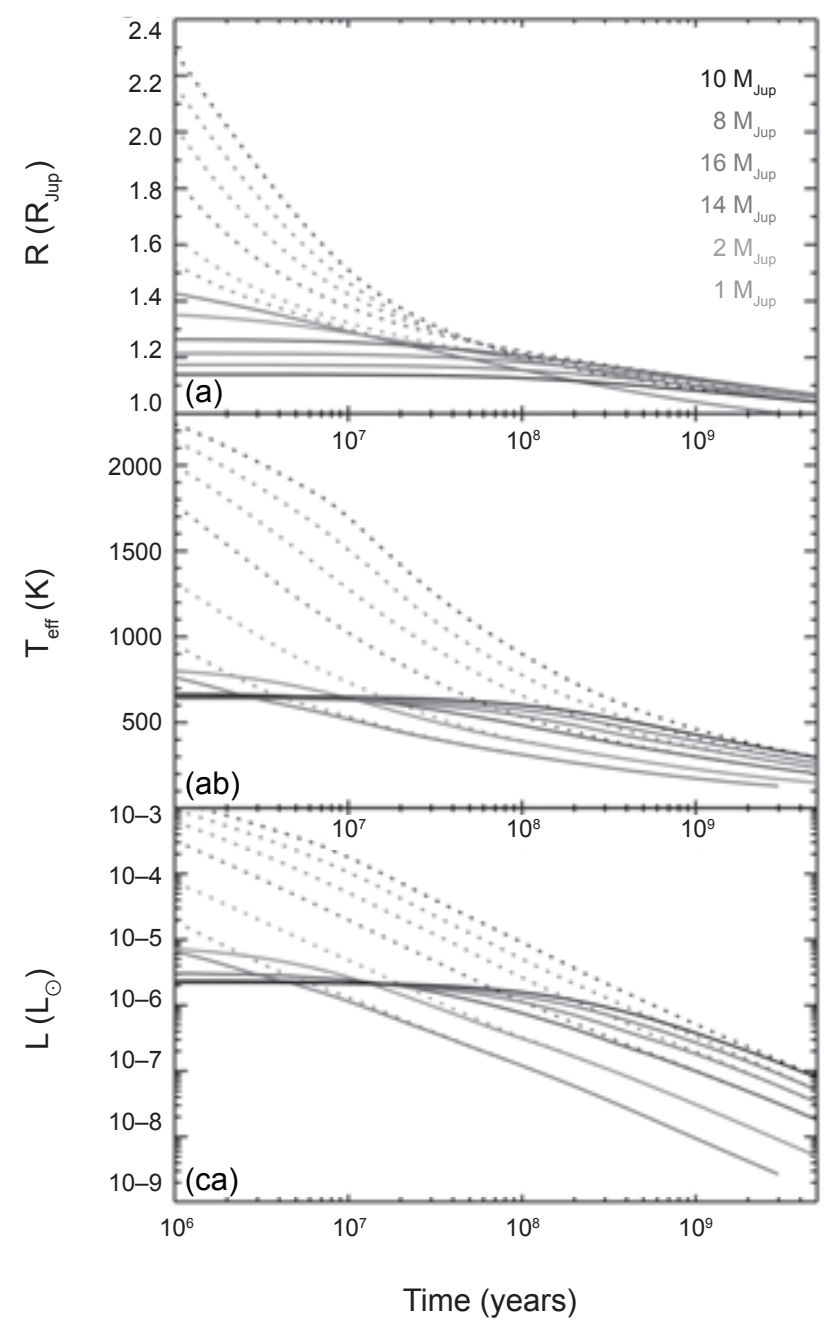

Fig. 5. Reproduction of Fig. 4 of Marley et al. (2007) showing the model radius, temperature, and luminosity of young Jupiters as a function of time since the beginning of their formation. Different colors reflect different planetary masses. Dotted lines indicate "hot start" planets, where adiabatic formation retains most of the initial energy and entropy; solid lines indicate "cold start," where accretion through a shock (as in the standard core accretion paradigm) results in loss of entropy. In either case, planets are significantly easier to detect at young ages.

The photometric detections of self-luminous planets have highlighted the complexities of modeling the atmospheres of these objects. Although they are similar to brown dwarfs, many of the directly imaged planets have temperatures that place them in the transitional region between clouddominated L dwarfs and methane-dominated T dwarfs - a change that is poorly understood even for the well-studied brown dwarfs. Cloud parameters in particular can make an enormous difference in estimates of properties like effective temperature and radius [see supplementary material in Marois et al. (2008) and subsequent discussion in Barman et al. (2011), Marley et al. (2012), Currie et al. (2011),

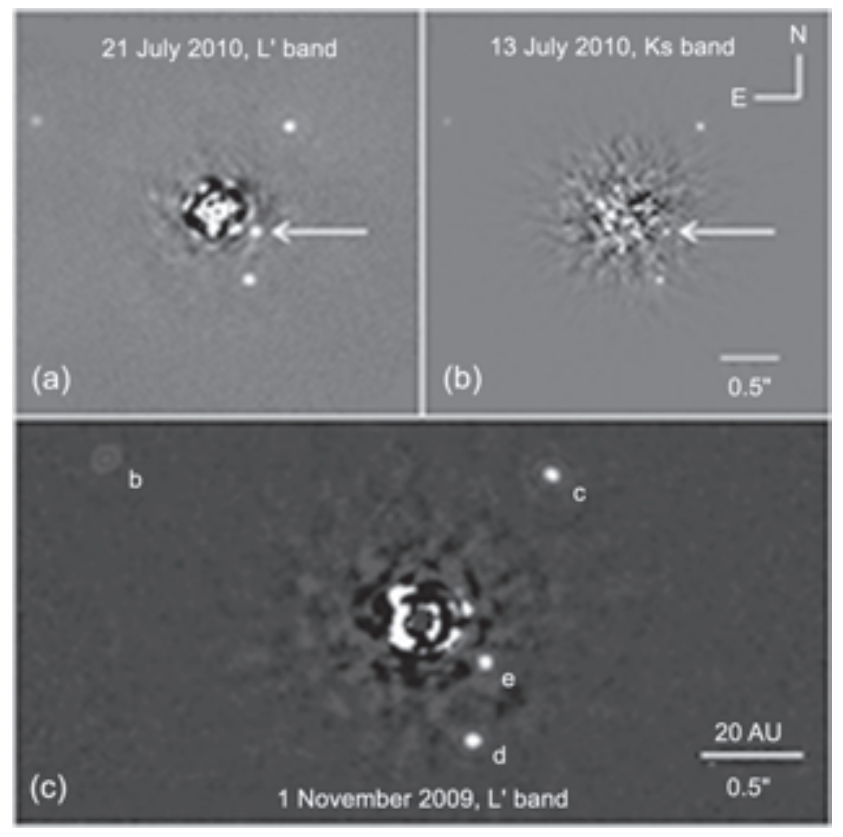

Fig. 6. Near-infrared Keck adaptive optics images of the HR 8799 system from Marois et al. (2010). Four giant planets, 3-7× the mass of Jupiter, are visible in near-infrared emission. The residual speckle pattern after PSF subtraction can be seen in the center of each image.

and discussion in the chapter by Madhusudhan et al. in this volume].

If a planet can be clearly resolved from its parent star, it is accessible not only through imaging but also spectroscopically. Integral field spectrographs are particularly well suited to this (e.g., Oppenheimer et al., 2013; Konopacky et al., 2013), since they also capture the spectrum of neighboring speckle artifacts, which can be used to estimate the speckle contamination of the planet itself. Spectra show that the self-luminous planets do (as expected) have low gravity and distinct atmospheric structure from brown dwarfs. In some cases, spectra have sufficiently high SNR that individual absorption features (e.g., of CO) can be clearly resolved (Konopacky et al., 2013), allowing direct measurements of atmospheric chemistry and abundances (Fig. 7).

\subsection{Future Groundbased and Spacebased Facilities}

Most direct imaging of exoplanets to date has taken place with traditional instruments attached to general-purpose AO systems, such as the Near InfraRed Camera (NIRC) 2 on the Keck II telescope or Nasmyth Adaptive Optics System (NACO) on the VLT. In fact, for most of these observations, the presence or absence of a coronagraph has had little effect on sensitivity, which is dominated by wavefront errors uncorrected by the AO system. Some sensitivity enhancement has come from dedicated exoplanet imaging cameras, employing techniques like dual-channel imaging, 


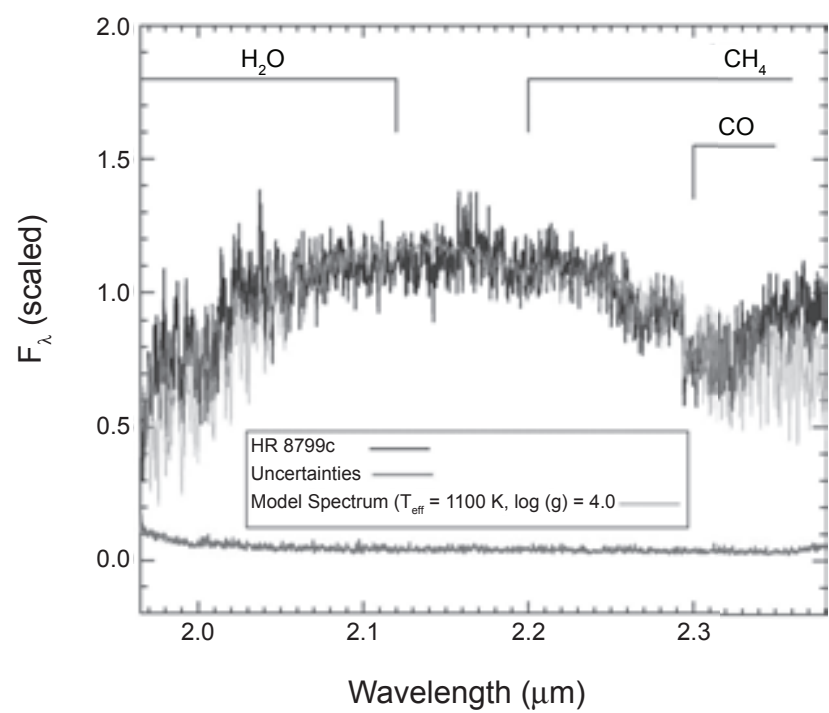

Fig. 7. High-resolution spectrum of the extrasolar planet HR 8799c taken with the OSIRIS spectrograph and the Keck adaptive optics system, reproduced from Konopacky et al. (2013). Residual speckle noise changes the overall spectral shape (e.g., the upturn at the long wavelength end) but does not inject narrow features; the $\mathrm{CO}$ break is clearly detected, as are many individual $\mathrm{CO}$ and $\mathrm{H}_{2} \mathrm{O}$ lines, while methane is absent.

in combination with conventional adaptive optics (Nielsen et al., 2013; Janson et al., 2013). The combination of pyramid wavefront sensing and adaptive secondary mirrors on the Large Binocular Telescope (LBT) and Magellan telescopes has shown excellent high-contrast performance (Skemer et al., 2012).

However, to significantly increase the number of imaged exoplanets will require dedicated instruments that combine very-high-performance adaptive optics, suitable coronagraphs, and exoplanet-optimized science instruments such as low-spectral-resolution diffraction-limited integral field spectrographs (IFS). The first such instrument to become operational is the Project 1640 coronagraphic IFS (Oppenheimer et al., 2013), integrated with a 3000-actuator AO system on the 5-m Hale telescope. The Subaru Coronagraphic Extreme AO System (SCExAO) (Martinache et al., 2012) is a 2000-actuator AO system that serves as a test bed for a wide variety of advanced technologies including focal-plane wavefront sensing and pupil-remapping coronagraphs. Finally, two facility-class planet imagers will be operational in 2014 on 8-m class telescopes: the Gemini Planet Imager (GPI) (Macintosh et al., 2012) and the VLT Spectro-Polarimetric High-contrast Exoplanet REsearch (SPHERE) facility (Beuzit et al., 2008; Petit et al., 2012). Both have 1500-actuator AO systems, apodized-pupil Lyot coronagraphs, and IFSs (SPHERE also incorporates a dualchannel IR imager and a high-precision optical polarimeter). Laboratory testing and simulations predict that they will achieve on-sky contrasts of better than $10^{6}$ at angles of
0.2 arcsec, although with the limitation of requiring bright stars ( $\mathrm{I}<8$ mag for GPI, $\mathrm{V}<12 \mathrm{mag}$ for SPHERE) to reach full performance. Both instruments will be located in the southern hemisphere, where the majority of young nearby stars are located. Simulated surveys (McBride et al., 2011) predict that GPI could discover $20-50$ jovian planets in a 900-hour survey.

Direct detection instruments have also been proposed for the upcoming 20-40-m Extremely Large Telescopes. These instruments exploit the large diameters of the telescope to achieve extremely small inner working angles $(0.03$ arcsec or less), opening up detection of protoplanets in nearby starforming regions orbiting at the snow line (Macintosh et al., 2006), or reflected light from mature giant planets close to their parent star (Kasper et al., 2010). At their theoretical performance limits, such telescopes could reach the contrast levels needed to detect rocky planets in the habitable zones of nearby $\mathrm{M}$ stars, although reaching that level may present insurmountable technical challenges. (Guyon et al., 2012).

A coronagraphic capability has been proposed for the 2.4-m Astrophysics Focused Telescope Assets (AFTA) Wide-Field Infrared Survey Telescope (WFIRST) mission (Spergel et al., 2013). Due to the obscured aperture and relative thermal stability of the telescope, it would likely be limited to contrasts of $10^{-9}$ at separations of 0.1 or 0.2 arcsec, but this would still enable a large amount of giant-planet and disk science, including spectral characterization of mature giant planets.

Direct detection of an Earth-analog planet orbiting a solar-type star, however, will almost certainly require a dedicated space telescope using either an advanced coronagraph - still equipped with adaptive optics - or a formation-flying starshade occulter.

\section{MICROLENSING}

\subsection{Planetary Microlensing}

7.1.1. Microlensing basics. A microlensing event occurs when two stars at different distances pass within $\sim 1$ mas of each other on the plane of the sky (Gaudi, 2012). Light from the source star "S" is bent by the lens star "L," so that the observer "O" sees the image "I" instead of the true source (see Fig. 8). If the source and the lens are perfectly aligned along the line of sight, the source is lensed into a ring (Chwolson, 1924; Einstein, 1936; Renn et al., 1997), called an Einstein ring, whose angular size is given by

$$
\theta_{\mathrm{E}}=\sqrt{\mathrm{\kappa M}_{\mathrm{L}} \pi_{\mathrm{rel}}} \sim 0.3 \text { mas }
$$

for typical values of the lens mass $\left(\mathrm{M}_{\mathrm{L}}=0.5 \mathrm{M}_{\odot}\right)$, lens distance $\left(D_{L}=6 \mathrm{kpc}\right)$, and source distance $\left(D_{S}=8 \mathrm{kpc}\right)$. In equation (4), $\pi_{\text {rel }}=\left(1 \mathrm{AU} / \mathrm{D}_{\mathrm{L}}\right)-\left(1 \mathrm{AU}=\mathrm{D}_{\mathrm{S}}\right)$ is the trigonometric parallax between the source and the lens, and $\mathrm{\kappa}=$ $8.14 \operatorname{mas~}_{\odot}^{-1}$.

If the source is offset from the lens by some small amount, it is lensed into two images that appear in line with 


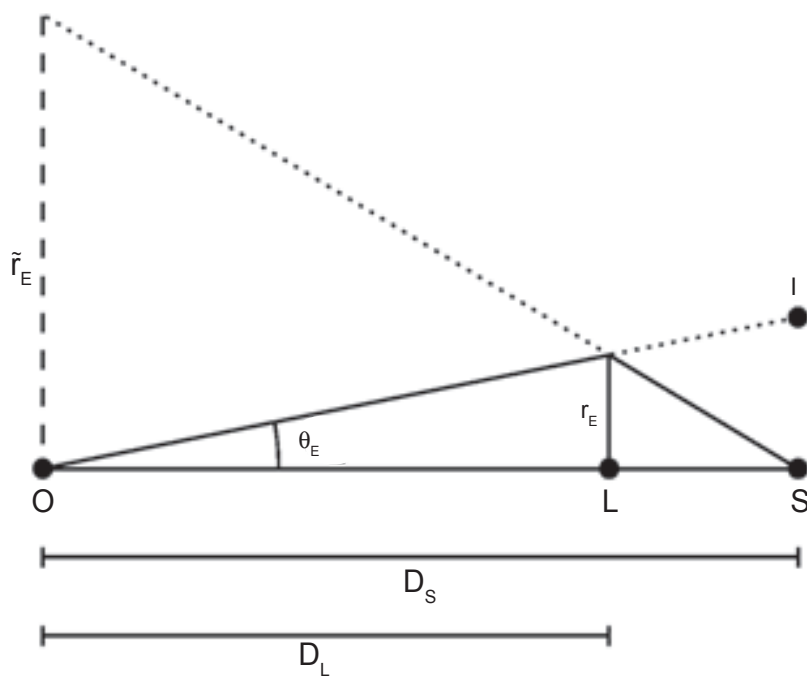

Fig. 8. Basic geometry of microlensing.

the source and the lens, and close to the Einstein ring as in Fig. 9. Because the size of the Einstein ring is so small, the two images of the source are unresolved and the primary observable is their combined magnification

$$
A=\frac{u^{2}+2}{u \sqrt{u^{2}+4}}
$$

where $\mathrm{u}$ is the projected separation between the source and the lens as a fraction of the Einstein ring. Since the source and the lens are both moving, $\mathrm{u}$ (and therefore A) is a function of time.

7.1.2. Types of planetary perturbations. If planets are gravitationally bound to a lensing star, the planet can be detected if one of the source images passes over or near the position of the planet. This creates a perturbation to the microlensing light curve of the host star. Because the images generally appear close to the Einstein ring, microlensing is most sensitive to planets with projected separations equal to the physical size of the Einstein ring in the lens plane, $\mathrm{r}_{\mathrm{E}}=\theta_{\mathrm{E}} \mathrm{D}_{\mathrm{L}}$.

Another way to think about this is to consider the magnification map. The magnification of the source by a point lens can be calculated for any position in space using equation (5), giving a radially symmetric magnification map. The source then traces a path across this map creating a microlensing event whose magnification changes as a function of time (and position). The presence of the planet distorts the magnification map of the lens and causes two or more caustics to appear, as shown by the gray curves in Fig. 10a. A perfect point source positioned at a point along the caustic curve will be infinitely magnified. In order to detect the planet, the source trajectory must pass over or near a caustic caused by the planet (Mao and Paczynski, 1991; Gould and Loeb, 1992; Griest and Safizadeh, 1998).

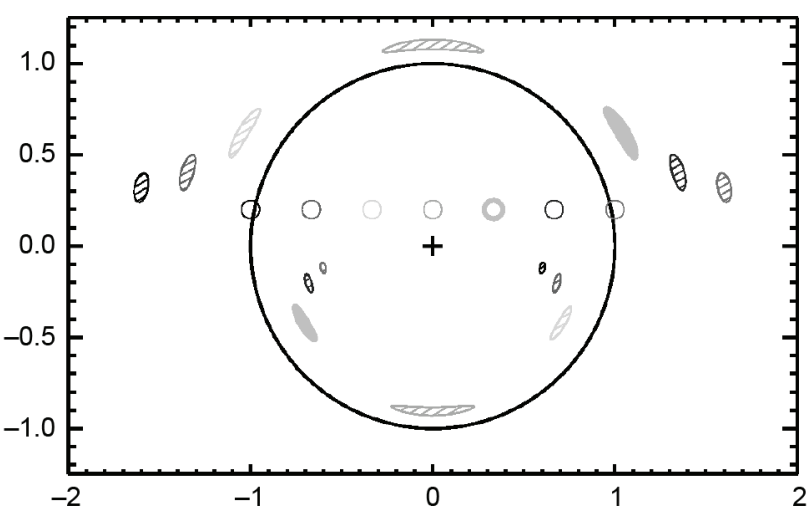

Fig. 9. Images of a lensed source star. The position of the source is indicated by the small circles. The filled voids show the lensed images for each source position. The large black circle shows the Einstein ring. The lens star is at the origin, marked by the plus.

There are two kinds of perturbations corresponding to the two sets of caustics produced by the planet. The "planetary caustic" is the larger caustic (or set of caustics) unassociated with the position of the lens star (right side of Fig. 10a). The "central caustic" is much smaller than the planetary caustic and is located at the position of the lens star (left side of Fig. 10a). Figure 10 shows two example source trajectories, their corresponding light curves, and details of the planetary perturbation in a planetary caustic crossing. As the mass ratio, $q$, decreases, so does the duration of the planetary perturbation. In addition, the detailed shape of the perturbation depends on the size of the source star relative to the size of the Einstein ring, $\rho$.

7.1.3. Planet masses from higher-order effects. The fundamental observable properties of the planet are the mass ratio between the planet and the lens star, $\mathrm{q}$, and the projected separation between the planet and the lens star as a fraction of the Einstein ring, s. Hence, while $\mathrm{q} \leq 10^{-3}$ definitively identifies the companion to the lens as a planet, its physical properties cannot be recovered without an estimate of $M_{L}$ and $D_{L}$. However, if $\theta_{E}$ and $\widetilde{r}_{E}$ (the size of the Einstein ring in the observer plane) can be measured, it is possible to obtain measurements of $\mathrm{M}_{\mathrm{L}}$ and $\mathrm{D}_{\mathrm{L}}$ (see Fig. 8), and hence the physical mass and projected separation of the planet: $\mathrm{m}_{\mathrm{p}}=\mathrm{qM}_{\mathrm{L}}$ and $\mathrm{a}_{\perp}=\mathrm{s} \theta_{\mathrm{E}} \mathrm{D}_{\mathrm{L}}$. These variables can be measured from higher-order effects in the microlensing light curve. If finite-source effects are observed (cf. Fig. 10e), $\theta_{\mathrm{E}}$ is measured since $\rho=\theta_{\star} / \theta_{\mathrm{E}}$ and the angular size of the source, $\theta_{\star}$, can be determined from the color-magnitude diagram (Yoo et al., 2004). Finally, as Earth orbits the Sun, the line of sight toward the event changes, giving rise to microlens parallax (Gould, 1992; Gould et al., 1994), allowing a measurement of $\widetilde{\mathrm{r}}_{\mathrm{E}}$

$$
\pi_{\mathrm{E}}=\frac{1 \mathrm{AU}}{\tilde{\mathrm{r}}_{\mathrm{E}}}=\frac{\pi_{\mathrm{rel}}}{\theta_{\mathrm{E}}}
$$



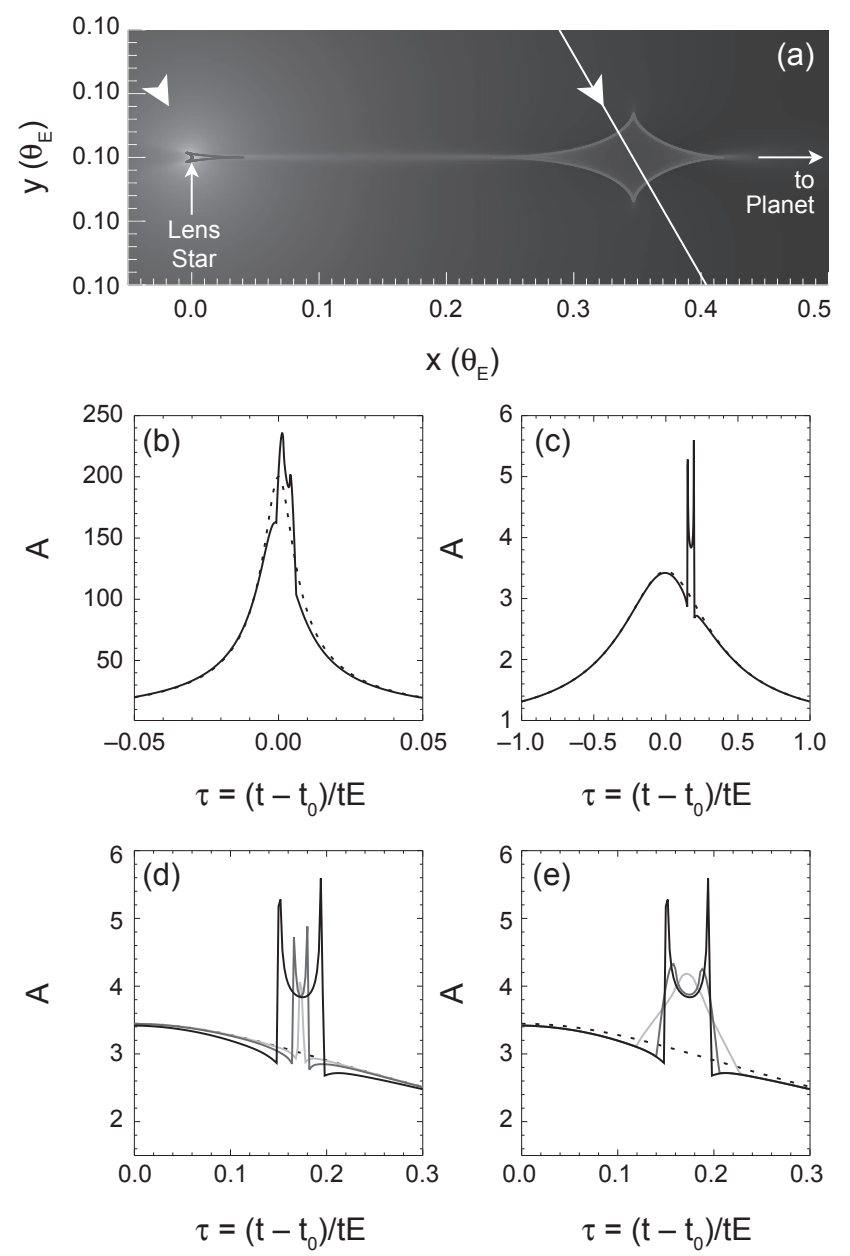

Fig. 10. (a) Magnification map for a planet with $q=0.001$ and $s=1.188$ and a source size $\rho=0.001$. The gray lines indicate the caustics. Two example source trajectories are shown. The scale is such that the Einstein ring is a circle of radius 1.0 centered at $(0,0)$. The planet is located at $(1.188,0)$, just outside the Einstein ring (off the righthand side of the plot). (b) Light curve corresponding to the lefthand source trajectory (a central caustic crossing). The dotted line shows the corresponding light curve for a point lens. (c) Light curve corresponding to the righthand source trajectory (a planetary caustic crossing). (d) Detail of (c) showing the variation in the planetary signal for different values of $q=10^{-3}, 10^{-4}$, and $10^{-5}$. (e) The variation in the planetary signal for different values of $\rho=0.001,0.01$, and 0.03 .

7.1.4. Microlensing degeneracies and false-positives. In microlensing the most common degeneracy is that planets with separation s produce nearly identical central caustics as planets with separation s ${ }^{-1}$ (Griest and Safizadeh, 1998). For planetary caustics, this is not a major problem since $\mathrm{s}$ (where $\mathrm{s}$ is larger than the Einstein ring) produces a "diamond"-shaped caustic, whereas $\mathrm{s}^{-1}$ produces a pair of "triangular" caustics (Gaudi and Gould, 1997). Additional degeneracies arise when higher-order effects such as parallax and the orbital motion of the lens are significant. In such cases, the exact orientation of the event on the sky becomes important and can lead to both discrete and continuous degeneracies in the relevant parameters (e.g., Gould, 2004; Skowron et al., 2011).

False positives are rare in microlensing events in which the source crosses a caustic. Because the magnification diverges at a caustic, this produces a discontinuity in the slope of the light curve, which is very distinctive (see Fig. 10). However, in events without caustic crossings, planetary signals can be mimicked by a binary source (Gaudi, 1998; Hwang et al., 2013), orbital motion of the lens (e.g., Albrow et al., 2000), or even starspots (e.g., Gould et al., 2013). Often multi-band data can help distinguish these scenarios, as in the case of starspots or lensing of two sources of different colors.

\subsection{Microlensing Observations in Practice}

The first microlensing searches were undertaken in the late 1980 s, primarily as a means to find massive compact halo objects [a dark matter candidate (Alcock et al., 1992; Aubourg et al., 1993)]. These searches were quickly expanded to include fields toward the galactic bulge to search for planets and measure the mass function of stars in the inner galaxy (Paczynski, 1991; Griest et al., 1991). One million stars must be observed to find one microlensing event, so the first surveys focused on simply detecting microlensing events. These surveys typically observed each field between once and a few times per night. However, the timescale of the planet is much shorter: a day or two for a Jupiter-mass planet down to an hour for an Earth-mass planet. Hence, follow-up groups target the known microlensing events to obtain the higher-cadence observations necessary to detect planets.

In practice, it is not possible to follow up all microlensing events, so the first priority is placed on the high-magnification events $(A \geq 50)$, i.e., the central caustic crossing events. Not only can the time of peak sensitivity to planets be predicted (around the time of maximum magnification), but these events are much more sensitive to planets than the average events, giving maximal planet-yield for the available resources (Griest and Safizadeh, 1998).

To date, almost 20 microlensing planets have been published, most of them found using the survey + follow-up method and in high-magnification events. Currently the main surveys for detecting microlensing events are the Optical Gravitational Lens Experiment (OGLE) (Udalski, 2003) and Microlensing Observations in Astrophysics (MOA) (Bond et al., 2004). Wise Observatory in Israel is also conducting a microlensing survey toward the bulge (Gorbikov et al., 2010; Shvartzvald and Maoz, 2012). These surveys combined now discover more than 2000 microlensing events each year. In addition, several groups are devoted to following up these events: the Microlensing Follow-Up Network ( $\mu$ FUN) (Gould et al., 2006), Microlensing Network for the Detection of Small Terrestrial Exoplanets (MiNDSTEp) (Dominik et al., 2010), Probing Lensing Anomalies NETwork (PLANET) (Beaulieu et al., 2006), and RoboNet (Tsapras et al., 2009). 


\subsection{Microlensing Planet Discoveries}

7.3.1. Highlights. 7.3.1.1. The first microlensing planet: The first microlensing planet, OGLE-2003-BLG-235/MOA2003-BLG-53Lb, was a 2.6-M $\mathrm{M}_{\text {Jup }}$ planet discovered in 2003 by the OGLE and MOA surveys (Bond et al., 2004). Although it was discovered and characterized by surveys, this planet was found in "follow-up mode" in which the MOA survey changed its observing strategy to follow this event more frequently once the planetary anomaly was detected.

7.3.1.2. Massive planets around M-dwarfs: Many of the planets discovered by microlensing have large mass ratios corresponding to jovian planets. At the same time, the microlensing host stars are generally expected to be $\mathrm{M}$ dwarfs, since those are the most common stars in the galaxy. Specifically, there are two confirmed examples of events for which the host star has been definitively identified to be an M dwarf hosting a super-Jupiter: OGLE-2005BLG-071 (Udalski et al., 2005; Dong et al., 2009) and MOA-2009-BLG-387 (Batista et al., 2011). The existence of such planets is difficult to explain since the coreaccretion theory of planet formation predicts that massive, jovian planets should be rare around $\mathrm{M}$ dwarfs (Laughlin et al., 2004; Ida and Lin, 2005). However, it is possible they formed through gravitational instability and migrated inward (Boss, 2006).

7.3.1.3. Multi-planet systems: Two of the microlensing events that host planets, OGLE-2006-BLG-109 (Gaudi et al., 2008) and OGLE-2012-BLG-0026 (Han et al., 2013), have signals from two different planets. The OGLE-2006BLG-109L system is actually a scale model of our solar system. The planets in this event are a Jupiter and a Saturn analog, with both planets at comparable distances to those planets around the Sun when the difference in the masses of the stars is taken into account.

7.3.1.4. Free-floating planets: Because microlensing does not require light to be detected from the lenses, it is uniquely sensitive to detecting free-floating planets. Since $\theta_{\mathrm{E}}$ scales as $\mathrm{M}^{1 / 2}$, free-floating planets have extremely small Einstein rings and hence give rise to short-duration events $(\leq 1 \mathrm{~d})$. Based on the analysis of several years of MOA survey data, Sumi et al. (2011) found that there are two free-floating Jupiters for every star.

7.3.2. The frequency of planets measured with microlensing. Figure 11 compares the sensitivity of microlensing to other techniques, where the semimajor axis has been scaled by the snow line, $\mathrm{a}_{\text {snow }}=2.7 \mathrm{AU}\left(\mathrm{M}_{\star} / \mathrm{M}_{\odot}\right)$. The "typical" microlensing host is an $\mathrm{M}$ dwarf rather than a $\mathrm{G}$ dwarf, so from the perspective of the core-accretion theory of planet formation, the relevant scales are all smaller. In this theory, the most important scale for giant planet formation is the location of the snow line, which depends on stellar mass (Ida and Lin, 2004). Microlensing is most sensitive to planets at $1 r_{E}$, which is roughly $3 \times a_{\text {snow }}$ for an $\mathrm{M}$ dwarf (i.e., $\mathrm{a}_{\text {snow }} \sim 1 \mathrm{AU}$ and $\mathrm{r}_{\mathrm{E}} \sim 3 \mathrm{AU}$ ).

The frequency, or occurrence rate, of planets can be calculated by comparing the sensitivities of individual

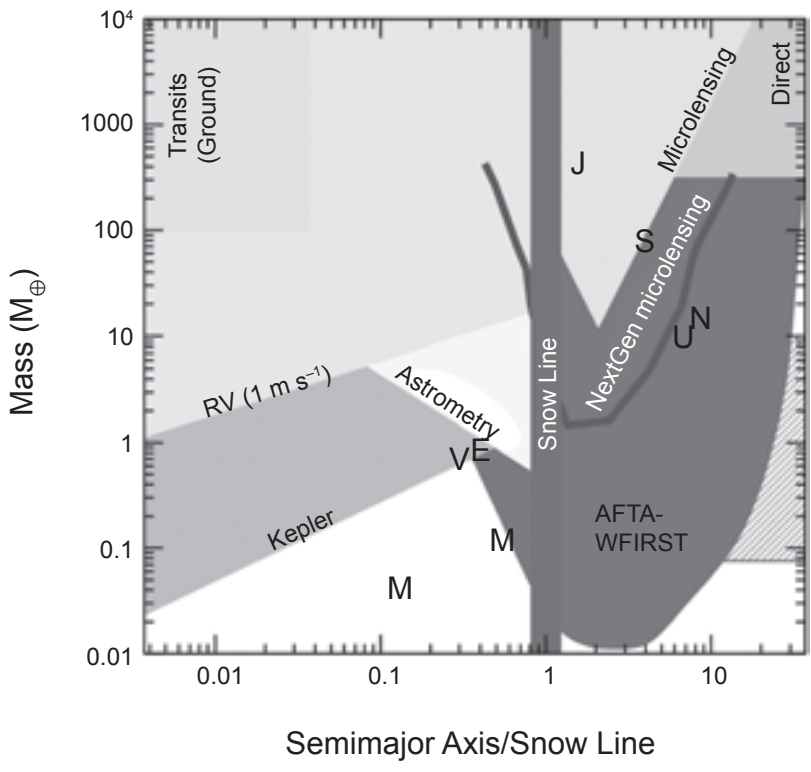

Fig. 11. Sensitivity of microlensing compared to other techniques. Figure courtesy of B. S. Gaudi and M. Penny.

events to the planets detected. Gould et al. (2010) analyzed high-magnification microlensing events observed by $\mu \mathrm{FUN}$ from 2005 to 2008 and found $\mathrm{dN} /(\mathrm{d} \log \mathrm{q} \mathrm{d} \log \mathrm{s})=$ $0.31 \pm 0.15$ planets per $\operatorname{dex}^{2}$ normalized at planets with Saturn-mass ratios. Cassan et al. (2012) also calculated the frequency of planets using events observed by PLANET, including both high- and low-magnification events. They found a similar planet frequency of $\mathrm{dN} /\left(\mathrm{d} \log\right.$ a $\left.\mathrm{d} \log \mathrm{m}_{\mathrm{p}}\right)=$ $10^{-0.62 \pm 0.22}\left(\mathrm{~m}_{\mathrm{p}} / \mathrm{M}_{\mathrm{Sat}}\right)^{0.73 \pm 0.17}$ normalized at Saturn masses and flat as a function of semimajor axis. Figures 8 and 9 in Gould et al. (2010) compare their result to the results from radial velocity for solar-type stars (Cumming et al., 2008; Mayor et al., 2009) and M dwarfs (Johnson et al., 2010b).

\subsection{The Future of Microlensing}

7.4.1. Second-generation microlensing surveys. Advances in camera technology now make it possible to carry out the ideal microlensing survey: one that is simultaneously able to monitor millions of stars while also attaining an $\sim 15$-min cadence. Both OGLE and MOA have recently upgraded to larger field-of-view cameras (Sato et al., 2008; Soszyński et al., 2012). They have teamed up with the Wise Observatory in Israel to continuously monitor a few of their fields (Shvartzvald and Maoz, 2012). In addition, the Korea Microlensing Telescope Network (KMTNet) (Park et al., 2012) is currently under construction. This network consists of three identical telescopes in Chile, Australia, and South Africa, which will conduct a high-cadence microlensing survey toward the galactic bulge. As these second-generation surveys get established, they will dominate the microlensing planet detections and the bulk of the detections will shift to planetary caustic crossings. Although high-magnification 
events are individually more sensitive to planets, they are very rare compared to low-magnification events. Hence, the larger cross-section of the planetary caustics will make low-magnification events the dominant channel for detecting planets in the new surveys.

7.4.2. Spacebased microlensing. The next frontier of microlensing is a spacebased survey, which has the advantages of improved photometric precision, the absence of weather, and better resolution. The improved resolution that can be achieved from space is a major advantage for characterizing the planets found by microlensing. In groundbased searches the stellar density in the bulge is so high that unrelated stars are often blended into the 1" PSF. This blending makes it impossible to accurately measure the flux from the lens star, and hence unless higher-order microlensing effects are observed, it is difficult to know anything about the lens. In space, it is possible to achieve a much higher resolution that resolves this blending issue, allowing an estimate of the lens mass based on its flux and hence a measurement of true planet masses rather than mass ratios.

The first microlensing survey satellite was proposed in Bennett and Rhie (2000, 2002). Currently, a microlensing survey for exoplanets has been proposed as a secondary science project for the Euclid mission (Penny et al., 2012; Beaulieu et al., 2013) and is a major component of the WFIRST mission (Spergel et al., 2013). The WFIRST mission is expected to detect thousands of exoplanets beyond the snow line (Spergel et al., 2013). The parameter space probed by this mission is complementary to that probed by the Kepler mission, which focused on detecting transits from close-in planets (see Fig. 11).

\section{ASTROMETRY}

\subsection{Introduction}

Steady advances in the eighteenth century improved the precision of stellar position measurements so that it was possible to measure the proper motions of stars, their parallax displacements due to Earth's motion around the Sun, and orbital motion caused by the gravitational tug of stellar companions (Perryman, 2012). While the impact of astrometry on exoplanet detection has so far been limited, the technique has enormous potential and is complementary to other methods (Gatewood, 1976; Black and Scargle, 1982; Sozzetti, 2005). Astrometry is most sensitive to wider orbits, because the center-of-mass displacement amplitude increases with orbital period. As a result, detectable orbital periods are typically several years. The need for measurement stability and precision over such long time baselines has been a challenging requirement for currently available instruments. Fortunately, with the successful launch of the Gaia satellite, the prospects for spacebased astrometric planet searches are good.

8.1.1. Parameterization of orbital motion. The term astrometry refers to the measurement of a star's position relative to the background sky, i.e., an astrometric orbit corresponds to the barycentric motion of a star caused by an invisible companion. This motion follows Kepler's laws and is parameterized by the period $\mathrm{P}$, the eccentricity e, the time of periastron passage $T_{0}$, its inclination relative to the sky plane $i$, the longitude of periastron $\omega$, the longitude of the ascending node $\Omega$, and the semimajor axis $\mathrm{a}_{1}$ expressed in angular units (Fig. 12). The Thiele-Innes constants A, B, $\mathrm{F}, \mathrm{G}$ are commonly used instead of the parameters $\mathrm{a}_{1}, \omega$, $\Omega$, i, because they linearize the orbit term in the general expression for an astrometric signal $\Lambda$ measured along an axis determined by the angle $\psi$

$$
\begin{aligned}
\Lambda= & \left(\Delta \alpha^{\star}+\mu_{\alpha^{\star}} \mathrm{t}\right) \cos \psi+\left(\Delta \delta+\mu_{\delta} \mathrm{t}\right) \sin \psi+\varpi \Pi_{\psi} \\
& +(\mathrm{BX}+\mathrm{GY}) \cos \psi+(\mathrm{AX}+\mathrm{FY}) \sin \psi
\end{aligned}
$$

where $\bar{\Phi}$ is the parallax, $\Pi_{\psi}$ is the parallax factor along $\psi$, $\mathrm{X}$ and $\mathrm{Y}$ are the rectangular coordinates (Hilditch, 2001)

$$
X=\cos E-e \quad Y=\sqrt{1-e^{2}} \sin E
$$

and $\mathrm{E}$ is the eccentric anomaly. This relation includes coordinate offsets in the equatorial system $\left(\Delta \alpha^{\star}, \Delta \delta\right)$, proper

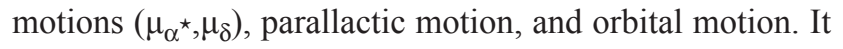
can be applied to both one- and two-dimensional measurements made by Hipparcos, Gaia, or interferometers.

8.1.2. Signal dependence on mass and distance. The semimajor axis $\bar{a}_{1}$ of a star's barycentric orbit is related to the stellar mass $\mathrm{m}_{1}$, the mass of the companion $\mathrm{m}_{2}$, and the orbital period by Kepler's third law (SI units)

$$
4 \pi^{2} \frac{\overline{\mathrm{a}}_{1}^{3}}{\mathrm{P}^{2}}=\mathrm{G} \frac{\mathrm{M}_{\mathrm{P}}^{3}}{\left(\mathrm{M}_{*}+\mathrm{M}_{\mathrm{P}}\right)^{2}}
$$

where $\mathrm{G}$ is the gravitational constant. The relation between angular and linear semimajor axes is proportional to the parallax, $a_{1} \propto \Phi \bar{a}_{1}$, thus the orbit's apparent angular size

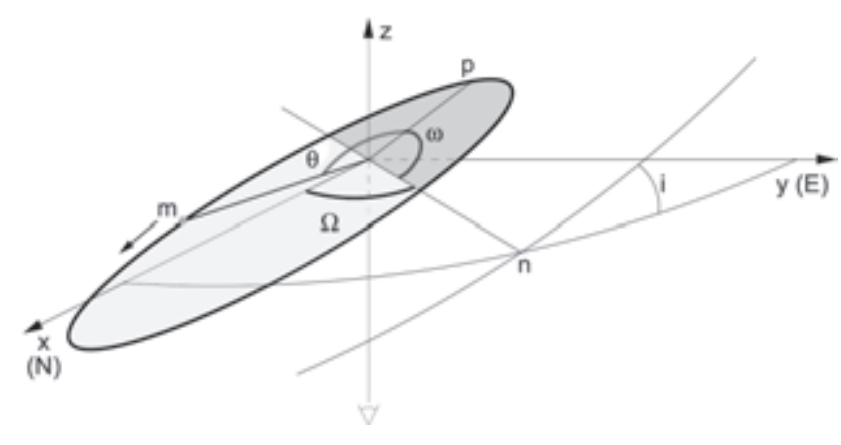

Fig. 12. Illustration of the orbit described by a star $(m)$ about the barycenter located at the origin. The observer sees the sky plane defined by the $x-y$ axes from below along the $z$ axis. The angles i, $\omega, \Omega, \Theta$, the ascending node $n$, and the periastron position $\mathrm{p}$ are indicated. By convention, $\mathrm{x}$ is north and y is east. Figure from Sahlmann (2012). 
decreases reciprocally with the system's distance from Earth. The value of $a_{1}$ determines the semi-amplitude of the periodic signal we intend to detect with astrometric measurements. Figure 13 shows the minimum astrometric signature $\mathrm{a}_{1, \min }$ derived from equation (9) for planets listed in the exoplanets.org database (Wright et al., 2011) on June 1, 2013, that have an entry for distance, star mass, orbital period, and planet mass, where we assumed circular orbits. For radial velocity planets, $\mathrm{a}_{1, \min }$ is a lower bound because we set $\sin i=1$. The spread at a given period originates in differing distances, star masses, and planet masses. Figure 13 illustrates the typical signal amplitudes for the known exoplanet population and highlights that only a small fraction of known planets are accessible with a measurement precision of 1 mas. It also shows that an improvement by only 1 order of magnitude in precision would set astrometry over the threshold of routine exoplanet detection.

8.1.3. Scientific potential. The motivation for using astrometry to carry out exoplanet searches is founded in the rich and complementary orbital information provided by this technique. Astrometric measurements determine the value of $\mathrm{m}_{2}^{3} /\left(\mathrm{m}_{1}+\mathrm{m}_{2}\right)^{2}$, thus if the host star mass is known, then planet mass $\mathrm{m}_{2}$ can be estimated without the sin $\mathrm{i}$ ambiguity of radial velocity measurements. An astrometric study of a statistical sample of exoplanets could therefore accurately determine the planet mass function and help to refine theories of planet formation. Equation (9) implies that any orbital configuration creates an astrometric signal and the amplitude increases with orbital period (see the trend in Fig. 13), making astrometry an ideal technique for the study of planets on long-period orbits. Because the technique measures the photocenter, it is sensitive to the detection of planets around fast-rotating stars with broad spectral lines or around very faint objects like brown dwarfs. There may also be a reduced sensitivity to stellar activity compared to radial velocity or photometric measurements (Eriksson and Lindegren, 2007; Lagrange et al., 2011). Since activity is currently hampering the detection of Earth-mass planets (e.g., Dumusque et al. 2012), astrometry may hold a distinct advantage for future searches, although the precision needed to detect Earth-like planets around the closest stars is at the level of $1 \mu \mathrm{arcsec}$. Astrometry is applicable to planet searches around nearby stars of various masses and ages, with benefits for the study of the planet mass function, of long-period planets, and of planets around active stars.

\subsection{Techniques and Instruments}

The precision $\sigma$ of an astrometric measurement is fundamentally limited by the ability to measure an image position on a detector. In the diffraction limit, it is therefore related to the wavelength $\lambda$, the aperture size $\mathrm{D}$, and the signalto-noise $\mathrm{S} / \mathrm{N}$, typically limited by photon noise $\mathrm{S} / \mathrm{N} \sim \sqrt{\mathrm{N}_{\mathrm{p}}}$

$$
\sigma \propto \frac{1}{\mathrm{~S} / \mathrm{N}} \frac{\lambda}{\mathrm{D}}
$$

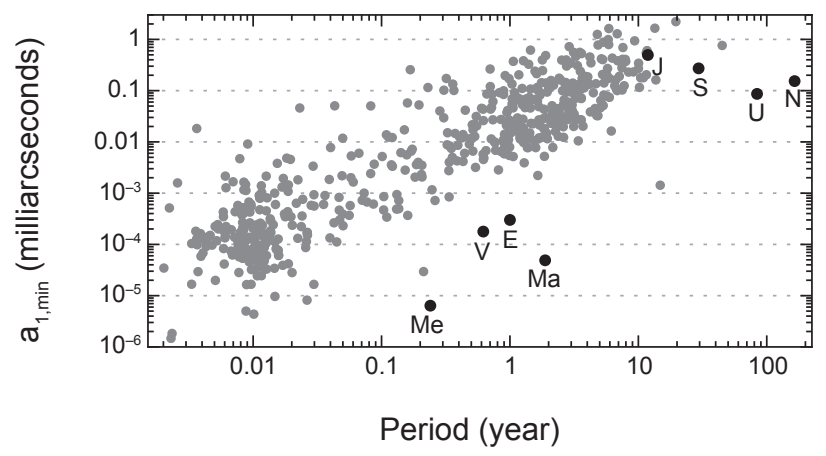

Fig. 13. Minimum astrometric signature of the host star as a function of orbital period for 570 planets (gray circles). For reference, the astrometric signatures of a solar-mass star located at a distance of $10 \mathrm{pc}$ caused by the solar system planets are shown with black circles and labeled with the planet initials.

thus, the achievable astrometric precision improves with the aperture size. For observations from the ground, the turbulence in Earth's atmosphere above the telescope is the dominant error source. It can be mitigated by modeling of seeing-limited observations (Lazorenko and Lazorenko, 2004), by the use of adaptive optics (Cameron et al., 2009), and with off-axis fringe tracking in dual-field interferometry (Shao and Colavita, 1992). Spaceborne instruments avoid atmospheric perturbations altogether and give access to nearly diffraction-limited observations, thus are ideal for high-precision astrometry work. Regardless of how the data were collected, the number of free astrometric parameters of a system with $\mathrm{n}$ planets is $5+\mathrm{n} \times 7$, i.e., at least 12 (see equation (7)), compared to $1+\mathrm{n} \times 5$ parameters for a radial velocity orbit adjustment (Wright and Howard, 2009). To obtain a robust solution and to minimize parameter correlations, e.g., between proper, parallactic, and orbital motion, a minimum timespan of one year and appropriate sampling of the orbital period are required.

8.2.1. Groundbased astrometry. Repeated imaging of a target and the measurement of its motion relative to background sources is a basic astrometric method, and several planet search surveys use seeing limited optical imaging with intermediate and large telescopes (Pravdo and Shaklan, 1996; Boss et al., 2009). Accuracies of better than 0.1 mas have been achieved with this method (Lazorenko et al., 2009), which satisfies the performance improvement necessary for efficient planet detection. Adaptive-optics assisted imaging is also being used, e.g., for a planet search targeting binaries with separations of a few arcseconds (Röll et al., 2011). An optical interferometer realizes a large effective aperture size by combining the light of multiple telescopes that translates into an achievable precision of 0.01 mas in the relative separation measurement of two stars typically less than $1^{\prime}$ apart (Shao and Colavita, 1992). Several observatories have implemented the necessary infrastructure and are pursuing astrometric planet search programs (Launhardt et al., 2008; Muterspaugh et al., 2010; Woillez et al., 2010; Sahlmann et 
al., 2013b). Similarly, very long baseline radio interferometry is a promising method for targeting nearby stars sufficiently bright at radio wavelengths (Bower et al., 2009).

8.2.2. Astrometry from space. Space astrometry was firmly established by the Hipparcos mission, which operated in 1989-1992 and resulted in the determination of positions, proper motions, and absolute parallaxes at the 1-mas level for 120,000 stars (Perryman et al., 1997). The satellite's telescope had a diameter of only $29 \mathrm{~cm}$ and scanned the entire celestial sphere several times to construct a global and absolute reference frame. However, Hipparcos data do not have the necessary precision to determine the astrometric orbits of the majority of known exoplanets. On a smaller scale but with slightly better precision, the Hubble Space Telescope's fine guidance sensor has made stellar parallax and orbit measurements possible (Benedict et al., 2001). Because the Stellar Interferometry Mission (Unwin et al., 2008) was discontinued, Gaia is the next space astrometry mission capable of detecting extrasolar planets.

\subsection{Results from Astrometry}

8.3.1. Combination with radial velocities. For a planet detected with RV, five out of seven orbital parameters are constrained. The two remaining parameters, the inclination $\mathrm{i}$ and $\Omega$, can be determined by measuring the astrometric orbit. The knowledge of the RV parameters (or the high weight of RV measurements) leads to a significant reduction of the required $\mathrm{S} / \mathrm{N}$ for a robust astrometric detection. Second, even an astrometric nondetection carries valuable information, e.g., an upper limit to the companion mass. Therefore, this type of combined analysis is so far the most successful application of astrometry in the exoplanet domain. Hipparcos astrometry yielded mass upper limits of RV planets (Perryman et al., 1996; Torres, 2007; Reffert and Quirrenbach, 2011) and revealed that, in rare cases, brown dwarfs (Sahlmann et al., 2011a) or stellar companions (Zucker and Mazeh, 2001) are mistaken for RV planets because their orbital planes are seen with small inclinations. Similarly, the Hubble fine guidance sensor was used to determine the orbits and masses of brown dwarf companions to Sun-like stars initially detected with RV (Martioli et al., 2010; Benedict et al., 2010) and groundbased imaging astrometry yielded a mass upper limit of $\sim 3.6 \mathrm{M}_{\text {Jup }}$ to the planet around GJ 317 (Anglada-Escudé et al., 2012). Sahlmann et al. (2011b) (Fig. 14) used Hipparcos data to eliminate low-inclination binary systems mimicking brown dwarf companions detected in a large RV survey, revealing a mass range where giant planets and close brown dwarf companions around Sun-like stars are extremely rare.

8.3.2. Independent discoveries. Working toward the goal of exoplanet detection, optical imaging surveys have succeeded in measuring the orbits of low-mass binaries and substellar companions to M dwarfs (Pravdo et al., 2005; Dahn et al., 2008), relying on astrometric measurements only. Interferometric observations revealed the signature of a Jupiter-mass planet around a star in an unresolved binary (Muterspaugh et al., 2010), which, if confirmed independently, represents the first planet discovered by astrometry. Recent improvements of imaging astrometry techniques toward 0.1-mas precision made the discovery of a $28-\mathrm{M}_{\text {Jup }}$ companion to an early L dwarf possible (Fig. 15) and demonstrated that such performance can be realized with a single-dish telescope from the ground.

\subsection{The Future}

Without a doubt, our expectations are high for the Gaia mission, which was launched on December 19, 2013. Gaia

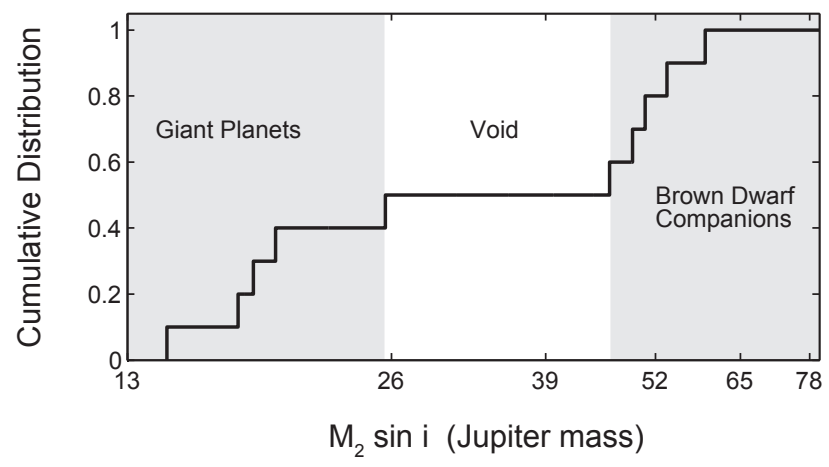

Fig. 14. The minimum mass distribution of substellar companions within $10 \mathrm{AU}$ of Sun-like stars from the Coralie RV survey after constraining the orbital inclinations with Hipparcos astrometry.

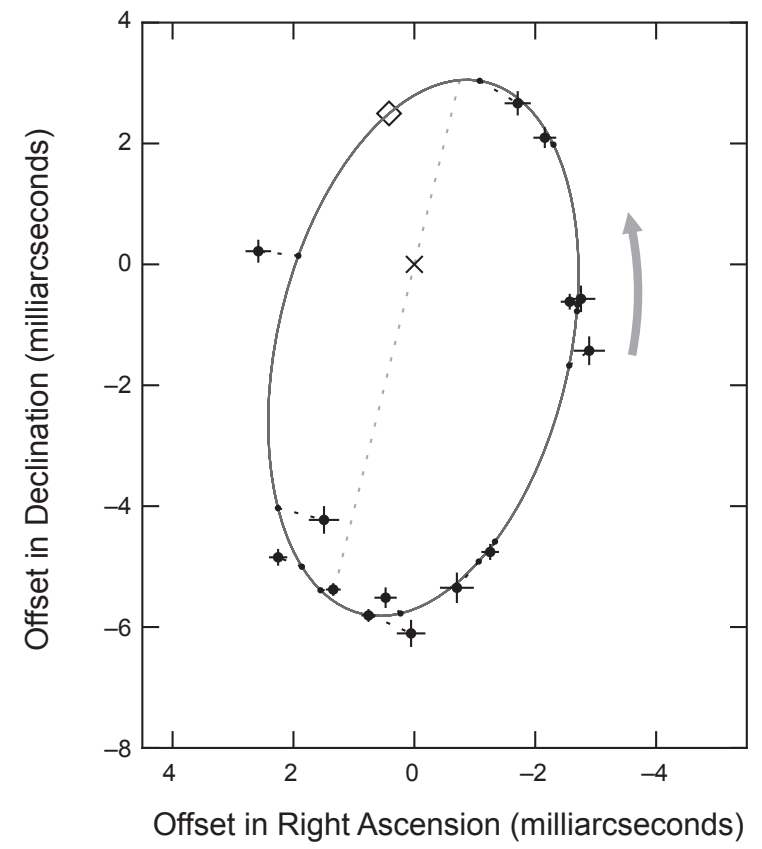

Fig. 15. The barycentric orbit of the L1.5 dwarf DENISP J082303.1-491201 caused by a $28-\mathrm{M}_{\text {Jup }}$ companion in a 246-day orbit discovered through groundbased astrometry with an optical camera on an 8-m telescope (Sahlmann et al., 2013a). 
is a cornerstone mission of the European Space Agency that will implement an all-sky survey of an estimated billion stellar objects with visible magnitudes of 6-20 (Perryman et al., 2001; de Bruijne, 2012). On average, the astrometry of a star will be measured 70 times over the mission lifetime of 5 years with a single measurement precision of $\sim 0.02-0.05$ mas for stars brighter than $\sim 14$ th magnitude. Another look at Fig. 13 shows that hundreds of known exoplanet systems will be detectable, and it is expected that Gaia will discover thousands of new exoplanets (Casertano et al., 2008), yielding a complete census of giant exoplanets in intermediate-period orbits around nearby stars. The sight of astrometric orbits caused by planets around stars will then become just as common as RV curves and dips in light curves are today. Assuming that it will perform as planned, Gaia will therefore add astrometry to the suite of efficient techniques for the study of exoplanet populations and will help us to advance our understanding of (exo)planet formation. It will also pave the way for future space astrometry missions aiming at detecting the Earth-like planets around nearby stars (Malbet et al., 2012).

At the same time, groundbased surveys will remain competitive because they offer long lifetimes, scheduling flexibility, and access to targets not otherwise observable. They are also necessary for technology development and demonstration. The upcoming generation of submillimeter/ optical interferometers and telescopes will have larger apertures and wide-field image correction, and hence provide us with even better astrometric performance and new opportunities for exoplanet science.

\section{STATISTICAL DISTRIBUTIONS OF EXOPLANET PROPERTIES}

In this section, we review and interpret the major statistical properties of extrasolar planets. We focus primarily on results from RV and transit surveys since they have produced the bulk of the discovered planets. Figure 16 shows known planets with measured masses and semimajor axes (projected for microlensing planets). The major archetypes of well-studied planets - cool Jupiters in 1-5-AU orbits, hot Jupiters in sub-0.1-AU orbits, and sub-Neptune-sized planets orbiting within $1 \mathrm{AU}$ - are all represented, although their relative frequencies are exaggerated due to differing survey sizes and yields. For more thorough reviews of exoplanet properties, the reader is directed to the literature (Howard, 2013; Cumming, 2011; Marcy et al., 2005; Udry and Santos, 2007).

\subsection{Abundant, Close-In Small Planets}

Planets intermediate in size between Earth and Neptune are surprisingly common in extrasolar systems, but notably absent in our solar system. The planet size and mass distributions (Fig. 17) demonstrate that small planets substantially outnumber large ones, at least for close-in orbits. Doppler surveys using the High Resolution Echelle
Spectrometer (HIRES) at Keck Observatory (Howard et al., 2010) and HARPS (Lovis et al., 2009; Mayor et al., 2011) at the 3.6-m ESO telescope have shown that small planets (Neptune-sized and smaller) significantly outnumber large ones for close-in orbits. Using the detected planets and detection completeness contours, the Eta-Earth Survey at Keck found that the probability of a star hosting a closein planet scales as $(\mathrm{M} \sin \mathrm{i})^{-0.48}$ : Small planets are more common. In absolute terms, $15 \%$ of Sun-like stars host one or more planets with $\mathrm{M} \sin \mathrm{i}=3-30 \mathrm{M}_{\oplus}$ orbiting within 0.25 AU. The HARPS survey confirmed the rising planet mass function with decreasing mass and extended it to $1-3-\mathrm{M}_{\oplus}$ planets. It also demonstrated that low-mass planets have small orbital eccentricities and are commonly found in multi-planet systems with two to four small planets orbiting the same star with orbital periods of weeks or months. It found that at least $50 \%$ of stars have one or more planets of any mass with $\mathrm{P}<100 \mathrm{~d}$.

The distribution of planet sizes (radii) measured by the Kepler mission (Fig. 17) follows the same qualitative trend as the mass distribution, with small planets being more common (Howard, 2013; Petigura et al., 2013; Fressin et al., 2013). However, the planet radius distribution extends with small error bars down to $1 \mathrm{R}_{\oplus}$ for close-in planets, while the mass distribution has $50 \%$ uncertainty level near $1 \mathrm{M}_{\oplus}$. The size distribution is characterized by a power-law rise in occurrence with decreasing size (Howard, 2013) down to a critical size of $\sim 2.8 \mathrm{R}_{\oplus}$, below which planet occurrence plateaus (Petigura et al., 2013). The small planets detected by Kepler $\left(<2 \mathrm{R}_{\oplus}\right)$ appear to have more circular orbits than

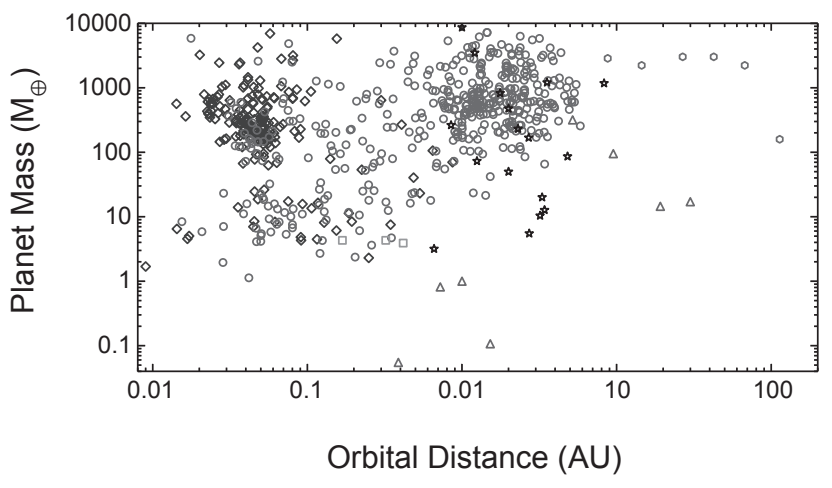

Fig. 16. Masses and orbital distances of planets from the Exoplanet Orbit Database (Wright et al., 2011) (exoplanets. org) as of July 2013. The recently discovered Earth-sized planet, Kepler-78b, is also included (Sanchis- Ojeda et al., 2013; Howard et al., 2013; Pepe et al., 2013). Extrasolar planets are coded according to their method of discovery: $\mathrm{RV}=$ circles, transit = diamonds, imaging = hexagons, gravitational microlensing $=$ stars, and pulsar timing $=$ squares. Planets in the solar system are triangles. Projected semimajor axis is plotted for microlensing planets while true semimajor axis is plotted for others. The occurrence of some planet types (e.g., hot Jupiters) are exaggerated relative to their true occurrence due to their relative ease of discovery. 
larger planets (Plavchan et al., 2012), suggesting reduced dynamical interactions.

The high occurrence of small planets with $\mathrm{P}<50 \mathrm{~d}$ likely extends to more distant orbits. As Kepler accumulates photometric data, it becomes sensitive to planets with smaller sizes and longer orbital periods. Based on 1.5 years of photometry, the small planet occurrence distribution as a
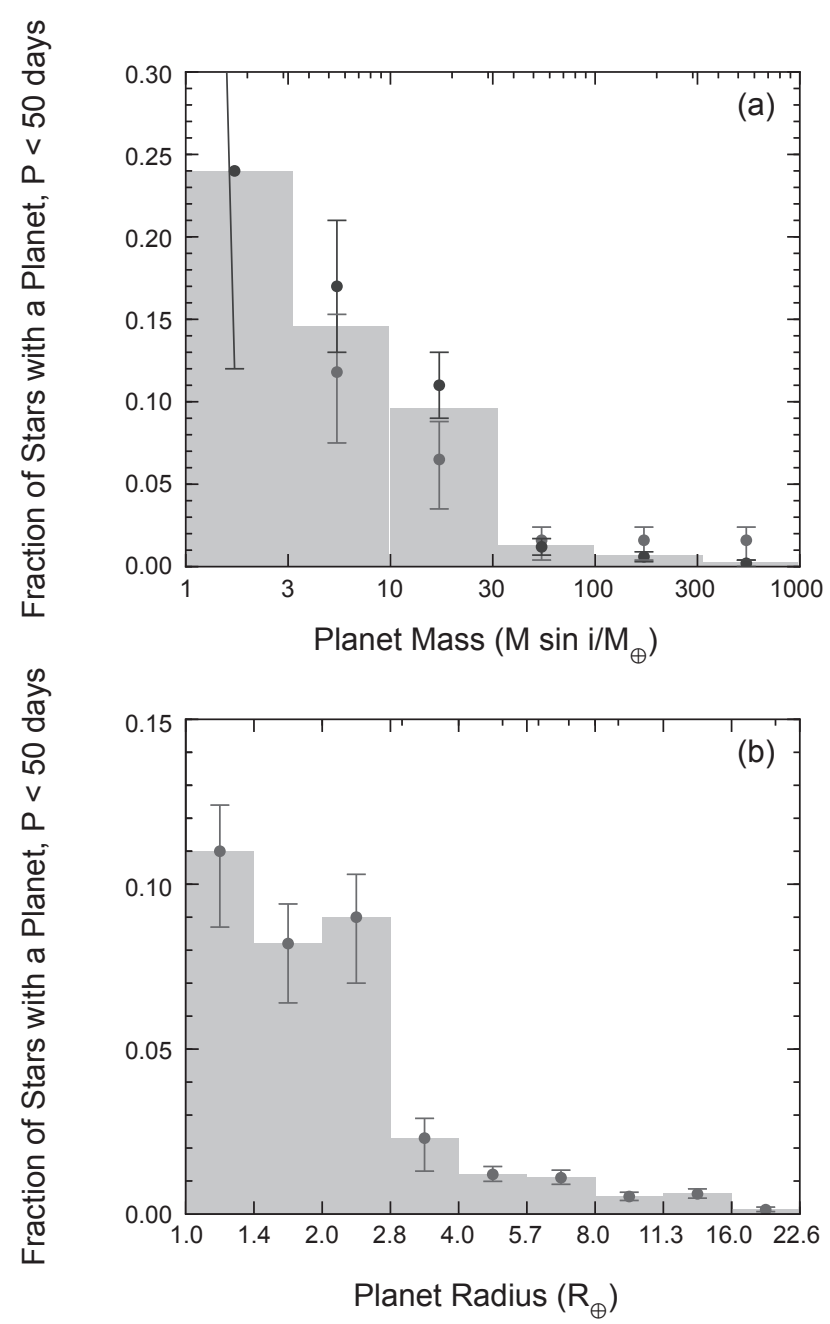

Fig. 17. The (a) mass and (b) size distributions of planets orbiting close to $\mathrm{G}$ and K-type stars. The distributions rise substantially with decreasing size and mass, indicating that small planets are more common than large ones. Planets smaller than $2.8 \mathrm{R}_{\oplus}$ or less massive than $30 \mathrm{M}_{\oplus}$ are found within $0.25 \mathrm{AU}$ of $30-50 \%$ of Sun-like stars. In (a), the size distribution is drawn from two studies of Kepler data: $\mathrm{Pe}$ tigura et al. (2013) for planets smaller than $4 \times$ Earth size and Howard et al. (2012) for larger planets. The mass (M sin i) distributions show the fraction of stars having at least one planet with an orbital period shorter than 50 days (orbiting inside $\sim 0.25 \mathrm{AU}$ ) are from separate Doppler surveys [darker lines = Howard et al. (2010); lighter lines = Mayor et al. (2011)], while the histogram shows their average values. Both distributions are corrected for survey incompleteness for small/low-mass planets to show the true occurrence of planets in nature. function of orbital period is flat to $\mathrm{P}=250 \mathrm{~d}$ (with higher uncertainty for larger P). Quantitatively, the mean number of planets per star per logarithmic period interval is proportional to $\mathrm{P}^{+0.11 \pm 0.05}$ and $\mathrm{P}^{-0.10 \pm 0.12}$ for $1-2-\mathrm{R}_{\oplus}$ and $2-4-\mathrm{R}_{\oplus}$ planets, respectively (Dong and $\mathrm{Zhu}, 2012$ ).

The Kepler planet distribution also shows that small planets are more abundant around around cool stars (Howard et al., 2012; although see Fressin et al., 2013, for an opposing view). M dwarfs observed by Kepler appear to have a high rate of overall planet occurrence, 0.9 planets per star in the size range $0.5-4 \mathrm{R}_{\oplus}$ in $\mathrm{P}<50 \mathrm{~d}$ orbits. Earth-sized planets $\left(0.5-1.4 \mathrm{R}_{\oplus}\right)$ are estimated to orbit in the habitable zones (HZ) of $15_{-6}^{+13 \%}$ of Kepler's M dwarfs (Dressing and Charbonneau, 2013). This estimate depends critically on the orbital bounds of the HZ; using more recent $\mathrm{HZ}$ models, the fraction of $\mathrm{M}$ dwarfs with Earth-sized planets in the HZ may be $3 \times$ higher (Kopparapu, 2013).

Of the Kepler planet host stars, $23 \%$ show evidence for two or more transiting planets. To be detected, planets in multi-transiting systems likely orbit in nearly the same plane, with mutual inclinations of a few degrees at most. The true number of planets per star (transiting or not) and their mutual inclinations can be estimated from simulated observations constrained by the number of single, double, triple, etc., transiting systems detected by Kepler (Lissauer et al., 2011b). Fang and Margot (2012) find an intrinsic multi-planet distribution with $54 \%, 27 \%, 13 \%, 5 \%$, and $2 \%$ of systems having $1,2,3,4$, and 5 planets with $\mathrm{P}<200 \mathrm{~d}$. Nearly all multi-planet systems $(85 \%)$ have mutual inclinations of less than $3^{\circ}$ (Fang and Margot, 2013; Johansen et al., 2012). Mutual inclinations of a few degrees are also suggested by comparison between the Kepler and HARPS data (Figueira et al., 2012). This high degree of co-planarity is consistent with planets forming in a protoplanetary disk without significant dynamical perturbations.

The ratios of orbital periods in multi-transiting systems provide additional dynamical constraints. These ratios are largely random (Fabrycky et al., 2012), with a modest excess just outside of period ratios that are consistent with dynamical resonances (ratios of 2.1, 3.2, etc.) and a compensating deficit inside (Lithwick and Wu, 2012). The period ratios of adjacent planet pairs demonstrate that $>31 \%,>35 \%$, and $>45 \%$ of two-planet, three-planet, and four-planet systems are dynamically packed; adding a hypothetical planet would gravitationally perturb the system into instability (Fang and Margot, 2013).

\subsection{Gas Giant Planets}

The orbits of giant planets are the easiest to detect using the Doppler technique and were the first to be studied statistically (e.g., Udry et al., 2003; Marcy et al., 2005). Observations over a decade of a volume-limited sample of $\sim 1000$ F-, G-, and K-type dwarf stars at Keck Observatory showed that $10.5 \%$ of G- and K-type dwarf stars host one or more giant planets $\left(0.3-10 \mathrm{M}_{\mathrm{Jup}}\right)$ with orbital periods of 2-2000 d (orbital distances of $\sim 0.03-3 \mathrm{AU}$ ). Within those 
parameter ranges, less-massive and more-distant giant planets are more common. Extrapolation of this model suggests that $17-20 \%$ of such stars have giant planets orbiting within $20 \mathrm{AU}(\mathrm{P}=90 \mathrm{yr}$ ) (Cumming et al., 2008). This extrapolation is consistent with a measurement of giant planet occurrence beyond 2 AU from microlensing surveys (Gould et al., 2010). However, the relatively few planet detections from direct imaging planet searches suggest that the extrapolation is not valid beyond $65 \mathrm{AU}$ (Nielsen and Close, 2010).

These smooth trends in giant planet occurrence mask pile-ups in semimajor axis (Wright et al., 2009). The orbital distances for giant planets show a preference for orbits larger than $\sim 1 \mathrm{AU}$ and to a lesser extent near $0.05 \mathrm{AU}$ ("hot Jupiters") (Fig. 18a). This period valley for apparently single planets is interpreted as a transition region between two categories of jovian planets with different migration histories (Udry et al., 2003). The excess of planets starting at $\sim 1$ AU approximately coincides with the location of the ice line, which provides additional solids that may speed the formation of planet cores or act as a migration trap for planets formed farther out (Ida and Lin, 2008). The semimajor axis distribution for giant planets in multi-planet systems is more uniform, with hot Jupiters nearly absent and a suppressed peak of planets in $>1$-AU orbits.

The giant planet eccentricity distribution (Fig. 18b) also differs between single- and multi-planet systems. The eccentricities of single planets can be reproduced by a dynamical model in which initially low eccentricities are excited by planet-planet scattering (Chatterjee et al., 2008). Multiplanet systems with a giant planet likely experienced

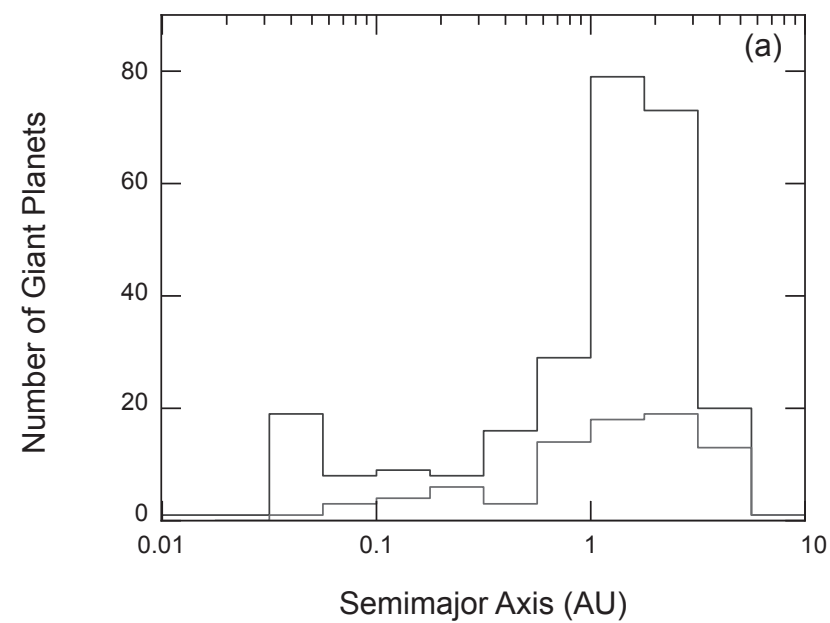

substantially fewer scattering events. The single-planet systems may represent the survivors of scattering events that ejected other planets in the system.

Metal-rich stars are more likely to host giant planets within 5 AU. This "planet-metallicity correlation" was validated statistically by Doppler surveys of stars with $\mathrm{M}_{\star}=0.7-1.2 \mathrm{M}_{\odot}$ and uniformly measured metallicities (Fischer and Valenti, 2005; Santos et al., 2004). The probability of a star hosting a giant planet is proportional to the square of the number of iron atoms in the star relative to the Sun, $\mathcal{P}$ (planet) $\propto \mathrm{N}_{\mathrm{Fe}}^{2}$. A later Doppler study spanned a wider range of stellar masses $\left(0.3-2.0 \mathrm{M}_{\odot}\right)$ and showed that the probability of a star hosting a giant planet correlates with both stellar metal content and stellar mass, $\mathcal{P}$ (planet) $\propto \mathrm{N}_{\mathrm{Fe}}^{1.2 \pm 0.2} \mathrm{M}_{\star}^{1.0 \pm 0.3}$ (Johnson et al., 2010a). Note that the planet-metallicity correlation only applies to gas giant planets. Planets larger than $4 \mathrm{R}_{\oplus}$ (Neptune-sized) preferentially orbit metal-rich stars, while smaller planets are are nondiscriminating in stellar metallicity (Buchhave et al., 2012). This pattern of host star metallicity can be explained if small planets commonly form in protoplanetary disks, but only a fraction of those small planets grow to a critical size in time to become gas giants.

Although hot Jupiters (giant planets with $\mathrm{P} \leq 10 \mathrm{~d}$ ) are found around only $0.5-1.0 \%$ of Sun-like stars (Wright et al., 2012), they are the most well-characterized planets because they are easy to detect and follow up with ground- and spacebased telescopes. However, their origin remains mysterious. In contrast to the commonly multiple sub-Neptunesized planets, hot Jupiters are usually the only detected

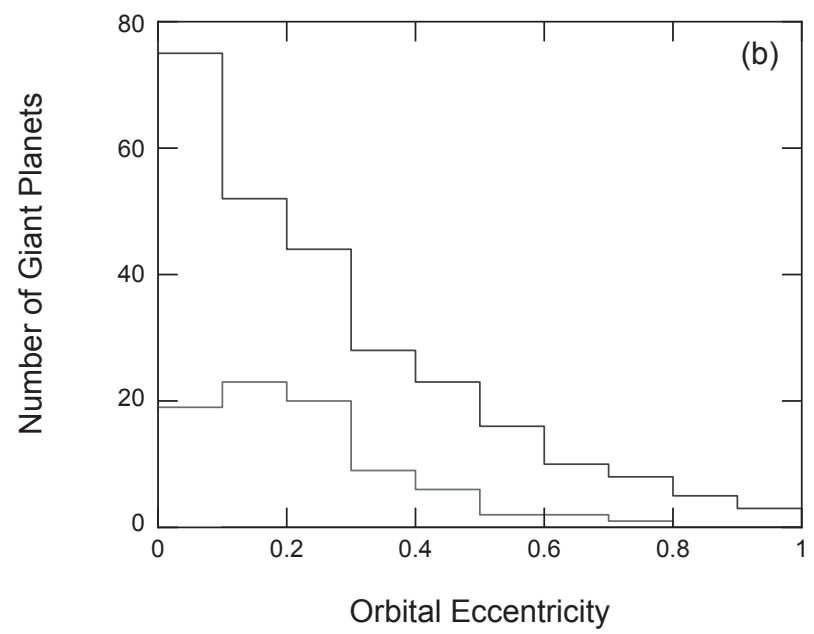

Fig. 18. Orbital characteristics of giant planets $\left(M_{P} \sin i>0.2 M_{\text {Jup }}\right)$ detected by Doppler surveys as cataloged on the Exoplanet Orbit Database (Wright et al., 2011). (a) The number distribution of semimajor axes shows that apparently single planets (top line) preferentially orbit at distances of $\sim 0.05 \mathrm{AU}$ and at $\sim 1-3 \mathrm{AU}$ from their host stars. These preferred orbits are diminished in multi-planet systems (bottom line). The decline in number of detected planets for orbits outside $\sim 3 \mathrm{AU}$ is not significant; fewer stars have been searched for such planets compared to the closer orbits. (b) The distribution of orbital eccentricities for apparently single planets (top line) span the full range, with low-eccentricity orbits being more common. Giant planets in multi-planet systems (bottom line) have orbits that are more commonly close to circular. The larger eccentricities of single planets suggests that they were dynamically excited from a quiescent, nearly circular origin, perhaps by planet-planet scattering that resulted in the ejection of all but one detectable planet per system. 
planet orbiting the host star within observational limits (Steffen et al., 2012). Many hot Jupiters have low eccentricities due to tidal circularization. The measured obliquities of stars hosting hot Jupiters display a peculiar pattern: Obliquities are apparently random above a critical stellar temperature of $\sim 6250 \mathrm{~K}$, but cooler systems are mostly aligned. In situ formation is unlikely for hot Jupiters because of insufficient protoplanetary disk mass so close to the star. It is more likely that they formed at several astronomical units, were gravitationally perturbed into orbits with random inclinations and high eccentricities, and were captured at $\sim 0.05$ AU by dissipation of orbital energy in tides raised on the planet. For systems with sufficiently strong tides raised by the planet on the star (which depend on a stellar convective zone that is only present below for $T_{\text {eff }} \leq 6250 \mathrm{~K}$ ), the stellar spin axis aligns to the orbital axis.

\subsection{Mass-Radius Relationships}

While the mass and size distributions provide valuable information about the relative occurrence of planets of different types, it remains challenging to connect the two. Knowing the mass of a planet only weakly specifies its size, and vice versa. This degeneracy can be lifted for $\sim 200$ planets with well-measured masses and radii (Fig. 19), most of which are transiting hot Jupiters. The cloud of points follows a diagonal band from low-mass/small-size to high-mass/ large-size. This band of allowable planet mass/size combinations has considerable breadth. Planets less massive than $\sim 30 \mathrm{M}_{\oplus}$ vary in size by a factor of $\sim 5$ and planets larger than $\sim 100 \mathrm{M}_{\oplus}$ (gas giants) vary by a factor of $\sim 2$. For the gas giants, the size dispersion at a given mass is due largely to two effects. First, planets in tight orbits receive higher stellar flux and are more commonly inflated. While higher stellar flux correlates with giant planet inflation (Weiss et al., 2013), it is unclear how the stellar energy is deposited in the planet's interior. Less importantly, the presence of a massive solid core (or distributed heavy elements) increases a planet's surface gravity, causing it to be more compact.

Low-mass planets show an even larger variation in size and composition. Three examples of sub-Neptune-sized planets illustrate the diversity. The planet Kepler-10b has a mass of $4.6 \mathrm{M}_{\oplus}$ and a density of $9 \mathrm{~g} \mathrm{~cm}^{-3}$, indicating a rock/ iron composition and no atmosphere (Batalha et al., 2011). In contrast, the planet Kepler-11e has a density of $0.5 \mathrm{~g} \mathrm{~cm}^{-3}$ and a mass of $8 \mathrm{M}_{\oplus}$. A substantial light-element atmosphere (probably hydrogen) is required to explain its mass and radius combination (Lissauer et al., 2011a). The masses and radii of intermediate planets lead to ambiguous conclusions about composition. For example, the bulk physical properties of GJ 1214b [6.5 $\mathrm{M}_{\oplus}, 2.7 \mathrm{R}_{\oplus}, 1.9 \mathrm{~g} \mathrm{~cm}^{-3}$ (Charbonneau et al., 2009)] are consistent with several compositions: a "super-Earth" with a rock/iron core surrounded by $\sim 3 \% \mathrm{H}_{2}$ gas by mass; a water-world planet consisting of a rock/iron core, water ocean, and atmosphere that contribute $\sim 50 \%$ of the mass; or a mini-Neptune composed of rock/iron, water, and $\mathrm{H} / \mathrm{He}$ gas (Rogers and Seager, 2010).

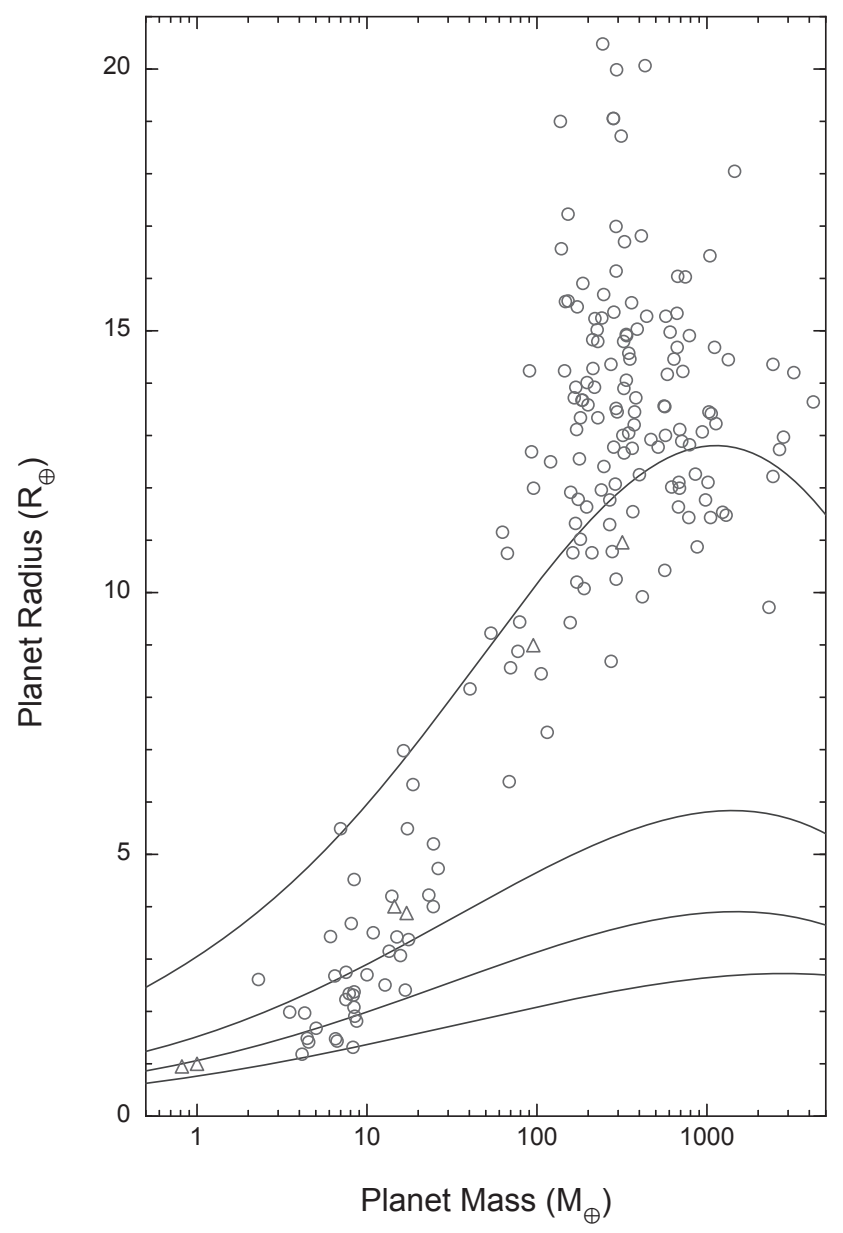

Fig. 19. Masses and radii of well-characterized planets from the Exoplanet Orbit Database (Wright et al., 2011). Extrasolar planets are shown as open circles and solar system planets are open triangles. Lines show model mass-radius relationships for idealized planets consisting of pure hydrogen (Seager et al., 2007), water, rock $\left(\mathrm{Mg}_{2} \mathrm{SiO}_{4}\right)$, or iron (Fortney et al., 2007). Poorly understood heating mechanisms inflate some gas giant planets (larger than $\sim 8 \mathrm{R}_{\oplus}$ ) to sizes larger than predicted by the simple hydrogen model. Smaller planets (less massive than $\sim 30 \mathrm{M}_{\oplus}$ ) show great diversity in size at a fixed mass, likely due to varying density of solids and atmospheric extent.

Acknowledgments. D.F. acknowledges support from NSF AST1207748 and NASA NNX12AC01C. A.W.H. acknowledges NASA grant NNX12AJ23G. Portions of this work performed under the auspices of the U.S. Department of Energy by Lawrence Livermore National Laboratory under Contract DEAC52-07NA27344. G.L. acknowledges support from NASA through Spitzer RSA 1438930. S.M. acknowledges support from National Space Foundation (NSF) grants AST-1006676, AST-1126413, AST-1310885, PSARC, the NASA Astrobiology Institute (NNA09DA76A), and the Center for Exoplanets and Habitable Worlds in the pursuit of precise RVs in the NIR. The Center for Exoplanets and Habitable Worlds is supported by the Pennsylvania State University, the Eberly College of Science, and the Pennsylvania Space Grant Consortium. Work by J.Y. 
was performed in part by a Distinguished University Fellowship from The Ohio State University and in part under contract with the California Institute of Technology (Caltech)/Jet Propulsion Laboratory (JPL) funded by NASA through the Sagan Fellowship Program executed by the NASA Exoplanet Science Institute.

\section{REFERENCES}

Agol E. et al. (2005) Mon. Not. R. Astron. Soc., 359, 567. Albrow M. D. et al. (2000) Astrophys. J., 534, 894.

Alcock C. et al. (1992) In Robotic Telescopes in the 1990s (A. V. Filippenko, ed.), pp. 193-202. ASP Conf. Ser. 34, Astronomical Society of the Pacific, San Francisco.

Alonso R. et al. (2004) Astrophys. J. Lett., 613, L153.

Anglada-Escudé G. et al. (2012) Astrophys. J., 746, 37.

Aubourg E. et al. (1993) The Messenger, 72, 20.

Baines E. K. et al. (2012) Astrophys. J., 761, 57.

Bakos G. Á. et al. (2007) Astrophys. J., 656, 552.

Bakos G. Á. et al. (2013) Publ. Astron. Soc. Pac., 125, 154.

Barbieri M. et al. (2007) Astron. Astrophys., 476, L13.

Barge P. et al. (2008) Astron. Astrophys., 482, L17.

Barman T. S. et al. (2011) Astrophys. J., 733, 65.

Batalha N. M. et al. (2010) Astrophys. J. Lett., 713, L103.

Batalha N. M. et al. (2011) Astrophys. J., 729, 27.

Batalha N. M. et al. (2013) Astrophys. J. Suppl., 204, 24.

Batista V. et al. (2011) Astron. Astrophys., 529, A102.

Batygin K. and Stevenson D. J. (2010) Astrophys. J. Lett., 714, L238.

Beaulieu J.-P. et al. (2006) Nature, 439, 437.

Beaulieu J. P. et al. (2013) ArXiv e-prints, arXiv:1303.6783.

Benedict G. F. et al. (2001) Astron. J., 121, 1607.

Benedict G. F. et al. (2010) Astron. J., 139, 1844.

Bennett D. P. and Rhie S. H. (2000) In Bull. Am. Astron. Soc., 32, 1053.

Bennett D. P. and Rhie S. H. (2002) Astrophys. J., 574, 985.

Beuzit J.-L. et al. (2008) In Ground-Based and Airborne Instrumentation for Astronomy II (I. S. McLean and M. M. Casali, eds.), SPIE Conf. Series 7014, Bellingham, Washington.

Black D. C. (1997) Astrophys. J. Lett., 490, L171.

Black D. C. and Scargle J. D. (1982) Astrophys. J., 263, 854.

Bond I. A. et al. (2004) Astrophys. J. Lett., 606, L155.

Bonfils X. et al. (2013) Astron. Astrophys., 549, A109.

Borucki W. J. et al. (2010) Science, 327, 977.

Borucki W. J. et al. (2012) Astrophys. J., 745, 120.

Borucki W. J. et al. (2013) Science, 340, 587.

Boss A. P. (2006) Astrophys. J., 643, 501.

Boss A. P. et al. (2009) Publ. Astron. Soc. Pac, 121, 1218.

Bower G. C. et al. (2009) Astrophys. J., 701, 1922.

Broeg C. et al. (2013) In EPJ Web Conf., 47, 3005.

Brogi M. et al. (2012) Nature, 486, 502.

Brogi M. et al. (2013) Astrophys. J., 767, 27.

Buchhave L. A. et al. (2012) Nature, 486, 375.

Butler R. P. et al. (1999) Astrophys. J., 526, 916.

Cameron P. B. et al. (2009) Astron. J., 137, 83.

Campbell B. et al. (1988) Astrophys. J., 331, 902.

Casertano S. et al. (2008) Astron. Astrophys., 482, 699.

Cassan A. et al. (2012) Nature, 481, 167.

Chabrier G. and Baraffe I. (2000) Annu. Rev. Astron. Astrophys., 38, 337. Charbonneau D. et al. (2000) Astrophys. J. Lett., 529, L45.

Charbonneau D. et al. (2007) In Protostars and Planets V (B. Reipurth et al., eds.), pp. 701-716. Univ. of Arizona, Tucson.

Charbonneau D. et al. (2009) Nature, 462, 891

Chatterjee S. et al. (2008) Astrophys. J., 686, 580.

Chauvin G. et al. (2004) Astron. Astrophys., 425, 2, L29.

Chauvin G. et al. (2010) Astron. Astrophys., 509, A52.

Chiang E. and Laughlin G. (2013) Mon. Not. R. Astron. Soc., 431, 3444.

Chwolson O. (1924) Astron. Nachr., 221, 329.

Collier Cameron A. et al. (2007) Mon. Not. R. Astron. Soc., 375, 951.

Collins K. A. et al. (2013) ArXiv e-prints, arXiv:1308.2296.

Crepp J. R. (2013) In AAS Meeting Abstracts, 221, 149.12.

Cumming A. (2011) In Exoplanets (S. Seager., ed.), pp. 191-214. Univ. of Arizona, Tucson.

Cumming A. et al. (2008) ArXiv e-prints, arXiv:0803.3357.

Currie T. et al. (2011) Astrophys. J., 729, 128.
Dahn C. C. et al. (2008) Astrophys. J., 686, 548. de Bruijne J. H. J. (2012) Astrophys. Space Sci., 341, 31. de Kok R. J. et al. (2013) Astron. Astrophys., 554, A82. Deming D. et al. (2009) Publ. Astron. Soc. Pac., 121, 952. Dominik M. et al. (2010) Astron. Nachr., 331, 671.

Dong S. and Zhu Z. (2012) ArXiv e-prints, arXiv:1212.4853.

Dong S. et al. (2009) Astrophys. J., 695, 970.

Dragomir D. et al. (2013) Astrophys. J. Lett., 772, L2.

Dressing C. D. and Charbonneau D. (2013) Astrophys. J., 767, 95.

Dumusque X. et al. (2012) Nature, 491, 207.

Einstein A. (1936) Science, 84, 506.

Eriksson U. and Lindegren L. (2007) Astron. Astrophys., 476, 1389.

Fabrycky D. C. and Murray-Clay R. A. (2010) Astrophys. J., 710, 1408.

Fabrycky D. C. et al. (2012) ArXiv e-prints, arXiv:1201.5415.

Fang J. and Margot J.-L. (2012) Astrophys. J., 761, 92.

Fang J. and Margot J.-L. (2013) Astrophys. J., 767, 115.

Figueira P. et al. (2012) Astron. Astrophys., 541, A139.

Fischer D. A. and Valenti J. (2005) Astrophys. J., 622, 1102.

Fischer D. A. et al. (2012) Mon. Not. R. Astron. Soc., 419, 2900.

Fischer D. A. et al. (2014) Astrophys. J., 210, 5.

Fortney J. J. et al. (2007) Astrophys. J., 659, 1661.

Fressin F. et al. (2011) Astrophys. J. Suppl., 197, 5.

Fressin F. et al. (2013) Astrophys. J., 766, 81.

Garcia-Melendo E. and McCullough P. R. (2009) Astrophys. J., 698, 558.

Gatewood G. (1976) Icarus, 27, 1

Gaudi B. S. (1998) Astrophys. J., 506, 533.

Gaudi B. S. (2012) Annu. Rev. Astron. Astrophys., 50, 411.

Gaudi B. S. and Gould A. (1997) Astrophys. J., 486, 85.

Gaudi B. S. et al. (2008) Science, 319, 927.

Ghasempour A. et al. (2012) In Modern Technologies in Space- and Ground-Based Telescopes and Instrumentation II (R. Navarro et al., eds.), SPIE Conf. Series 8450, Bellingham, Washington.

Gillon M. et al. (2007) Astron. Astrophys., 472, L13.

Gorbikov E. et al. (2010) Astrophys. Space Sci., 326, 203.

Gould A. (1992) Astrophys. J., 392, 442.

Gould A. (2004) Astrophys. J., 606, 319.

Gould A. and Loeb A. (1992) Astrophys. J., 396, 104.

Gould A. et al. (1994) Astrophys. J. Lett., 423, L105.

Gould A. et al. (2006) Astrophys. J. Lett., 644, L37.

Gould A. et al. (2010) Astrophys. J., 720, 1073.

Gould A. et al. (2013) Astrophys. J., 763, 141.

Gray D. F. (1997) Nature, 385, 795.

Griest K. and Safizadeh N. (1998) Astrophys. J., 500, 37.

Griest K. et al. (1991) Astrophys. J. Lett., 372, L79.

Guyon O. et al. (2005) Astrophy. J., 622, 1, 744.

Guyon O. et al. (2006) Astrophys. J. Suppl. Ser., 167, 81.

Guyon O. et al. (2012) In Adaptive Optics Systems III (B. L. Ellerbroek et al., eds.), SPIE Conf. Series 8447, Bellingham, Washington.

Halverson S. et al. (2012) In Ground-Based and Airborne Instrumentation for Astronomy IV (I. S. McLean et al., eds.), pp. 84468Q-84468Q-16. SPIE Conf. Series 8446, Bellingham, Washington.

Han C. et al. (2013) Astrophys. J. Lett., 762, L28.

Hatzes A. P. et al. (1997) Astrophys. J., 478, 374.

Hatzes A. P. et al. (2003) Astrophys. J., 599, 1383.

Henry G. W. et al. (2000) Astrophys. J. Lett., 529, L41.

Hilditch R.W. (2001) An Introduction to Close Binary Stars. Cambridge Univ., Cambridge.

Holman M. J. and Murray N. W. (2005) Science, 307, 1288.

Holman M. J. et al. (2010) Science, 330, 51.

Horne K. (2003) In Scientific Frontiers in Research on Extrasolar Planets (D. Deming and S. Seager, eds.), pp. 361-370. ASP Conf. Ser. 294, Astronomical Society of the Pacific, San Francisco.

Howard A. W. (2013) Science, 340, 572.

Howard A. W. et al. (2010) Science, 330, 653.

Howard A. W. et al. (2012) Astrophys. J. Suppl., 201, 15.

Howard A. W. et al. (2013) Nature, 503, 381.

Hunter T. R. and Ramsey L. W. (1992) Publ. Astron. Soc. Pac., 104, 1244.

Hwang K.-H. et al. (2013) Astrophys. J., 778, 55.

Ida S. and Lin D. N. C. (2004) Astrophys. J., 604, 388.

Ida S. and Lin D. N. C. (2005) Astrophys. J., 626, 1045.

Ida S. and Lin D. N. C. (2008) Astrophys. J., 673, 487.

Janson M. et al. (2013) Astrophys. J., 773(1), 73.

Jenkins J. M. et al. (2010) Astrophys. J. Lett., 713, L87.

Johansen A. et al. (2012) Astrophys. J., 758, 39.

Johnson J. A. et al. (2009) Astrophys. J. Lett., 692, L100. 
Johnson J. A. et al. (2010a) Publ. Astron. Soc. Pac, 122, 905. Johnson J. A. et al. (2010b) Publ. Astron. Soc. Pac., 122, 149.

Kalas P. et al. (2008) Science, 322, 1345.

Kalas P. et al. (2013) Astrophys. J., 775, 56.

Kasdin N. J. et al. (2003) Astrophys. J., 582, 2, 1147.

Kasper M. et al. (2010) In Ground-Based and Airborne Instrumentation for Astronomy III (I. S. McLean et al., eds.), SPIE Conf. Series 7735 , Bellingham, Washington.

Konacki M. et al. (2003) Astrophys. J., 597, 1076.

Konopacky Q. M. et al. (2013) Science, 339, 6126, 1398.

Kopparapu R. K. (2013) Astrophys. J. Lett., 767, L8.

Kuchner M. J. and Traub W. A. (2002) Astrophy. J., 570, 2, 900.

Kuzuhara M. et al. (2013) Astrophys. J., 774, 11.

Lafrenière D. et al. (2007a) Astrophys. J., 660, 770.

Lafrenière D. et al. (2007b) Astrophys. J., 670, 1367.

Lafrenière D. et al. (2010) Astrophys. J., 719, 497.

Lagrange A.-M. et al. (2010) Science, 329, 57.

Lagrange A.-M. et al. (2011) Astron. Astrophys., 528, L9.

Latham D. W. et al. (1989) Nature, 339, 38.

Laughlin G. et al. (2004) Astrophys. J. Lett., 612, L73.

Launhardt R. et al. (2008) In Optical and Infrared Interferometry (M. Schöller et al., eds.), SPIE Conf. Series 7013, Bellingham, Washington.

Lazorenko P. F. and Lazorenko G. A. (2004) Astron. Astrophys., 427, 1127.

Lazorenko P. F. et al. (2009) Astron. Astrophys., 505, 903.

Léger A. et al. (2009) Astron. Astrophys., 506, 287.

Lintott C. J. et al. (2013) Astron. J., 145, 151.

Lissauer J. J. et al. (2011a) Nature, 470, 53.

Lissauer J. J. et al. (2011b) Astrophys. J. Suppl., 197, 8.

Lithwick Y. and Wu Y. (2012) Astrophys. J. Lett., 756, L11.

Lithwick Y. et al. (2012) Astrophys. J., 761, 122.

Liu M. C. et al. (2010) In Adaptive Optics Systems II (B. L. Ellerbroek et al., eds.), SPIE Conf. Series 7736, Bellingham, Washington.

Lockwood A. C. et al. (2014) ArXiv e-prints, arXiv:1402.0846.

Lovis C. and Fischer D. (2011) In Exoplanets (S. Seager, ed.), pp. 27-53. Univ. of Arizona, Tucson.

Lovis C. et al. (2009) In Transiting Planets (F. Pont et al., eds.), pp. 502-505. IAU Symp. 253, Cambridge Univ., Cambridge.

Lyot B. (1939) Mon. Not. R. Astron. Soc., 99, 580.

Macintosh B. et al. (2006) In Advances in Adaptive Optics II (B. L. Ellerbroek and D. B. Calia, eds.), SPIE Conf. Series 6272, Bellingham, Washington.

Macintosh B. A. et al. (2012) In Ground-Based and Airborne Instrumentation for Astronomy IV (I. S. McLean et al., eds.), pp. 84461U-84461U-9. SPIE Conf. Series 8446, Bellingham, Washington.

Mahadevan S. et al. (2012) In Ground-Based and Airborne Instrumentation for Astronomy IV (I. S. McLean et al., eds.), SPIE Conf. Series 8446, Bellingham, Washington.

Mahmud N. I. et al. (2011) Astrophys. J., 736, 123.

Malbet F. et al. (2012) Exp. Astron., 34, 385.

Mannings V. et al., eds. (2000) Protostars and Planets IV. Univ. of Arizona, Tucson. 1422 pp.

Mao S. and Paczynski B. (1991) Astrophys. J. Lett., 374, L37.

Marcy G. et al. (2005) Progr. Theor. Phys. Suppl., 158, 24.

Marley M. S. et al. (2007) Astrophys. J., 655, 541.

Marley M. S. et al. (2012) Astrophys. J., 754, 135.

Marmier M. et al. (2013) Astron. Astrophys., 551, A90.

Marois C. et al. (2006) Astrophys. J., 641, 556.

Marois C. et al. (2008) Science, 322, 1348.

Marois C. et al. (2010) Nature, 468, 1080.

Martinache F. et al. (2012) In Adaptive Optics Systems III (B. L. Ellerbroek et al., eds.), pp. 84471Y-84471Y-8. SPIE Conf. Series 8447, Bellingham, Washington.

Martioli E. et al. (2010) Astrophys. J., 708, 625.

Mayor M. and Queloz D. (1995) Nature, 378, 355.

Mayor M. et al. (2003) The Messenger, 114, 20.

Mayor M. et al. (2009) Astron. Astrophys., 493, 639.

Mayor M. et al. (2011) ArXiV e-prints, arXiv:1109.2497.

McBride J. et al. (2011) Publ. Astron. Soc. Pac., 123(904), 692.

McCullough P. R. et al. (2006) Astrophys. J., 648, 1228.

Moorhead A. V. et al. (2011) Astrophys. J. Suppl., 197, 1.

Morton T. D. and Johnson J. A. (2011) Astrophys. J., 738, 170.

Muterspaugh M. W. et al. (2010) Astron. J., 140, 1657.

Nersisyan S. R. et al. (2013) Optics Express, 21(7), 8205.

Nielsen E. L. and Close L. M. (2010) Astrophys. J., 717, 878.
Nielsen E. L. et al. (2013) Astrophys. J., 776(1), 4.

Oppenheimer B. R. et al. (2013) Astrophys. J., 768(1), 24.

Paczynski B. (1991) Astrophys. J. Lett., 371, L63.

Park B.-G. et al. (2012) In Ground-Based and Airborne

Telescopes IV (L. M. Stepp et al., eds.), SPIE Conf.

Series 8444, Bellingham, Washington.

Penny M. T. et al. (2012) ArXiv e-prints, arXiv:1206.5296.

Pepe F. et al. (2011) Astron. Astrophys., 534, A58.

Pepe F. et al. (2013) Nature, 503, 377.

Perrin M. D. et al. (2003) Astrophys. J., 596, 702.

Perryman M. (2012) EPJ H, 37, 745.

Perryman M. A. C. et al. (1996) Astron. Astrophys., 310, L21.

Perryman M. A. C. et al. (1997) Astron. Astrophys., 323, L49.

Perryman M. A. C. et al. (2001) Astron. Astrophys., 369, 339.

Petigura E. A. et al. (2013) Astrophys. J., 770, 69.

Petit C. et al. (2012) In Adaptive Optics Systems III (B. L. Ellerbroek et al., eds.), pp. 84471Z-84471Z-12. SPIE Conf. Series 8447, Bellingham, Washington.

Plavchan P. et al. (2012) ArXiv e-prints, arXiv:1203.1887.

Pravdo S. H. and Shaklan S. B. (1996) Astrophys. J., 465, 264.

Pravdo S. H. et al. (2005) Astrophys. J., 630, 528.

Quirrenbach A. et al. (2012) In Ground-Based and Airborne Instrumentation for Astronomy IV (I. S. McLean et al., eds.), SPIE Conf. Series 8446, Bellingham, Washington.

Ramsey L. W. et al. (2008) Publ. Astron. Soc. Pac., 120, 887.

Redman S. L. et al. (2011) Astrophys. J. Suppl., 195, 24

Redman S. L. et al. (2012) Astrophys. J. Suppl., 199, 2.

Reffert S. and Quirrenbach A. (2011) Astron. Astrophys., 527, A140.

Reipurth B. et al., eds. (2007) Protostars and Planets V. Univ. of Arizona, Tucson. 1024 pp.

Renn J. et al. (1997) Science, 275, 184.

Ricker G. R. et al. (2010) Bull Am. Astron. Soc., 42, 450.06.

Rodler F. et al. (2012) Astrophys. J. Lett., 753, L25.

Rogers L. A. and Seager S. (2010) Astrophys. J., 716, 1208.

Röll T. et al. (2011) In GAIA: At the Frontiers of Astrometry (C. Turon et al., eds.), pp. 429-432. EAS Publ. Ser., Vol. 45, Cambridge Univ., Cambridge.

Sahlmann J. (2012) Observing exoplanet populations with high-precision astrometry, Ph.D. thesis, Observatoire de Genève, Université de Genève.

Sahlmann J. et al. (2011a) Astron. Astrophys., 528, L8.

Sahlmann J. et al. (2011b) Astron. Astrophys., 525, A95.

Sahlmann J. et al. (2013a) ArXiv e-prints, arXiv:1306.3225.

Sahlmann J. et al. (2013b) Astron. Astrophys., 551, A52.

Sanchis-Ojeda R. et al. (2013) Astrophys. J., 774, 54.

Santerne A. et al. (2013) ArXiv e-prints, arXiv:1310.0748.

Santos N. C. et al. (2004) Astron. Astrophys., 415, 1153.

Sato T. et al. (2008) Exp. Astron., 22, 51.

Schwab C. et al. (2012) ArXiv e-prints, arXiv:1212.4867.

Schwamb M. E. et al. (2013) Astrophys. J., 768, 127.

Seager S. and Deming D. (2010) Annu. Rev. Astron. Astrophys., 48, 631.

Seager S. et al. (2007) Astrophys. J., 669, 1279.

Shao M. and Colavita M. M. (1992) Annu. Rev. Astron. Astrophys., 30, 457.

Shvartzvald Y. and Maoz D. (2012) Mon. Not. R. Astron. Soc., $419,3631$.

Sivaramakrishnan A. et al. (2001) Astrophys. J., 552(1), 397.

Skemer A. J. et al. (2012) Astrophys. J., 753, 14

Skowron J. et al. (2011) Astrophys. J., 738, 87.

Snellen I. A. G. et al. (2013) Astrophys. J., 764, 182.

Soszyński I. et al. (2012) Acta Astron., 62, 219.

Soummer R. et al. (2011) Astrophys. J., 729, 144.

Sozzetti A. (2005) Publ. Astron. Soc. Pac., 117, 1021.

Spanò P. et al. (2012) In Ground-Based and Airborne Instrumentation for Astronomy IV (I. S. McLean et al., eds.), SPIE Conf. Series 8446, Bellingham, Washington.

Spergel D. et al. (2013) ArXiv e-prints, arXiv:1305.5425.

Steffen J. H. et al. (2012) Proc. Natl. Acad. Sci., 109, 7982.

Strassmeier K. G. et al. (2008) In Ground-Based and Airborne Instrumentation for Astronomy II (I. S. McLean and M. M. Casali, eds.), SPIE Conf. Series 7014, Bellingham, Washington.

Sumi T. et al. (2011) Nature, 473, 349.

Torres G. (2007) Astrophys. J. Lett., 671, L65.

Torres G. et al. (2004) Astrophys. J., 614, 979.

Tsapras Y. et al. (2009) Astron. Nachr, 330, 4.

Udalski A. (2003) Acta Astron., 53, 291. 
Udalski A. et al. (2005) Astrophys. J. Lett., 628, L109.

Udry S. and Santos N. C. (2007) Annu. Rev. Astron. Astrophys., 45, 397.

Udry S. et al. (2003) Astron. Astrophys., 407, 369.

Unwin S. C. et al. (2008) Publ. Astron. Soc. Pac., 120, 38.

Weiss L. M. et al. (2013) Astrophys. J., 768, 14.

Winn J. N. et al. (2011) Astrophys. J. Lett., 737, L18.

Woillez J. et al. (2010) In Optical and Infrared Interferometry II (W. C. Danchi et al., eds), p. 7734-12. SPIE Conf. Series 7734, Bellingham, Washington.
Wright J. T. and Howard A. W. (2009) Astrophys. J. Suppl., 182, 205.

Wright J. T. et al. (2009) Astrophys. J., 693, 1084.

Wright J. T. et al. (2011) Publ. Astron. Soc. Pac., 123, 412.

Wright J. T. et al. (2012) Astrophys. J., 753, 160

Ycas G. et al. (2012a) Optics Lett., 37, 2199.

Ycas G. G. et al. (2012b) Optics Express, 20, 6631.

Yoo J. et al. (2004) Astrophys. J., 603, 139.

Zucker S. and Mazeh T. (2001) Astrophys. J., 562, 549.

Zuckerman B. and Song I. (2004) Annu. Rev. Astron. Astrophys., 42, 685. 